\title{
Legendre-Gauss collocation methods for nonlinear neutral delay differential equations
}

Jingjun Zhao, Yang Cao and Yang $\mathrm{Xu}^{*}$

\section{${ }^{*}$ Correspondence:} yangx@hit.edu.cn Department of Mathematics, Harbin Institute of Technology, Harbin, 150001, China

\begin{abstract}
In this paper, an efficient Legendre-Gauss collocation method is introduced for solving nonlinear neutral delay differential equations (NDDEs). Firstly, the single-step Legendre-Gauss collocation method is presented for NDDEs; we analyze the convergence of the method with different delay functions. Then the multi-domain Legendre-Gauss collocation method is presented, which is based on the single-step one; the results of convergence are also obtained. In addition, numerical results are presented to confirm our analysis.
\end{abstract}

Keywords: Legendre-Gauss collocation methods; nonlinear neutral delay differential equations; spectral accuracy

\section{Introduction}

Delay differential equations (DDEs) arise in a variety of fields such as biology, economics, control theory, and so on (see [1-5]). In particular, neutral delay differential equations (NDDEs) provide an important mathematical instrument to model several electromagnetic problems [6].

Recently, much literature has been devoted to the numerical solutions of NDDEs (see [7-10]). Meanwhile, a Legendre-tau method was proposed and analyzed for linear DDEs with one constant delay in [11]. The Legendre-Gauss collocation methods were studied for ordinary differential equations (ODEs) based on the Legendre polynomial expansions in $[12,13]$. Moreover, the Legendre-Gauss collocation methods were developed for nonlinear DDEs in [14]. Wang and Guo proposed an efficient numerical integration process for initial value problems of first order ODEs, based on the Legendre-Gauss-Radau interpolation, which is easy to implement and possesses the spectral accuracy in [15]. However, it is more interesting but challenging to develop and analyze the type of high-order methods for nonlinear NDDEs of the form

$$
\left\{\begin{array}{l}
U^{\prime}(t)=f\left(t, U(t), W(t), \frac{d}{d t} W(t)\right), \quad 0 \leq t \leq T, \\
U(t)=V(t), \quad t \leq 0,
\end{array}\right.
$$

where $W(t)=U(\theta(t)), f, \theta$, and $V$ are given functions.

If the initial function $V(t)$ does not link smoothly with the solution $U(t)$ at 0 , this discontinuity point is spread forward on a set of primary discontinuity points where the solution, unlike the non-neutral case, remains solely of class $C^{0}[16]$.

C) 2015 Zhao et al.; licensee Springer. This is an Open Access article distributed under the terms of the Creative Commons Attribution License (http://creativecommons.org/licenses/by/4.0), which permits unrestricted use, distribution, and reproduction in any medium, provided the original work is properly credited. 
The question is crucial of how to deal with the primary discontinuity points. Firstly, we assume that $T$ is very small such that there is no primary discontinuity point on $[0, T]$, then a single-step scheme for (1) is constructed. The scheme has an infinite order of accuracy both in time and delay variable. On the basis of the single-step scheme, we partition $[0, T]$ into several subintervals, and construct multi-domain scheme on $[0, T]$, on which there are primary discontinuity points. The multi-domain scheme enjoys remarkable advantages over the Runge-Kutta type methods, since the approximate solution of $U(t)$ is $C^{\infty}$-continuous in the interior of each subinterval if $f, \theta, V$ are $C^{\infty}$-continuous [16].

The paper is organized as follows. In Section 2, we present and analyze the single-step Legendre-Gauss collocation method with three different delay terms $\theta(t)$ on $[0, T]$, where $T$ is small enough, and we provide some numerical results to justify our theoretical analysis. In Section 3, the multi-domain version is described on $[0, T]$, on which there exist primary discontinuity points, and the convergence results are also derived. Some numerical results justify our theoretical analysis.

\section{The single-step Legendre-Gauss collocation method}

In this section, we describe and analyze a single-step numerical process for the NDDE (1) using the Legendre-Gauss interpolation when there is no discontinuous point on $[0, T]$. The single-step scheme serves as a base for the multi-domain one to be presented in the forthcoming section, which is suitable for the situation with discontinuous points.

\subsection{Preliminaries}

Let $L_{l}(t)$ be the standard Legendre polynomial of degree $l$. The shifted Legendre polynomials are defined by (see [12])

$$
L_{T, l}(t)=L_{l}\left(\frac{2 t}{T}-1\right)=\frac{(-1)^{l}}{l !} \partial_{t}^{l}\left(t^{l}\left(1-\frac{t}{T}\right)^{l}\right), \quad l=0,1,2, \ldots
$$

According to the properties of the standard Legendre polynomials, we have

$$
\begin{aligned}
& (l+1) L_{T, l+1}(t)-(2 l+1)\left(\frac{2 t}{T}-1\right) L_{T, l}(t)+l L_{T, l-1}(t)=0, \quad l \geq 1, \\
& \frac{d}{d t} L_{T, l+1}(t)-\frac{d}{d t} L_{T, l-1}(t)=\frac{2}{T}(2 l+1) L_{T, l}(t), \quad l \geq 1, \\
& \left|L_{T, l}(t)\right| \leq 1, \quad t \in[0, T], l \geq 1 .
\end{aligned}
$$

The set of $L_{T, l}(t)$ is a complete $L^{2}(0, T)$-orthogonal system, namely, $\int_{0}^{T} L_{T, l}(t) L_{T, m}(t) d t=$ $\frac{T}{2 l+1} \delta_{l, m}$, where $\delta_{l, m}$ is the Kronecker symbol. Thus, for any $v \in L^{2}(0, T), v(t)=\sum_{l=0}^{\infty} \hat{v}_{T, l} L_{T, l}$ and $\hat{v}_{T, l}=\frac{2 l+1}{T} \int_{0}^{T} v(t) L_{T, l}(t) d t$.

Now, we introduce the shifted Legendre-Gauss interpolation. Denote the nodes of the standard Legendre-Gauss interpolation on the interval $[-1,1)$ by $t_{j}^{N}(0 \leq j \leq N)$. In particular, $t_{0}^{N}=-1$. The corresponding Christoffel numbers are $\omega_{j}^{N}(0 \leq j \leq N)$. The nodes of the shifted Legendre-Gauss interpolation on the interval $[0, T)$ are the distinct zeros of $L_{T, N}(t)+L_{T, N+1}(t)$, denoted by $t_{T, j}^{N}(0 \leq j \leq N)$. Clearly, $t_{T, j}^{N}=\frac{T}{2}\left(t_{j}^{N}+1\right)$. The corresponding Christoffel numbers are $\omega_{T, j}^{N}=\frac{T}{2} \omega_{j}^{N}(0 \leq j \leq N)$. 
Let $\mathcal{P}_{N}(0, T)$ be the set of polynomials of degree at most $N$. Due to the property of the standard Legendre-Gauss quadrature, it follows that, for any $\phi \in \mathcal{P}_{2 N}(0, T)$, one has

$$
\int_{0}^{T} \phi(t) d t=\frac{T}{2} \sum_{j=0}^{N} \omega_{j}^{N} \phi\left(\frac{T}{2}\left(t_{j}^{N}+1\right)\right)=\sum_{j=0}^{N} \omega_{T, j}^{N} \phi\left(t_{T, j}^{N}\right) .
$$

Let $(u, v)_{T}$ and $\|v\|_{T}$ be the inner product and the norm of space $L^{2}(0, T)$, respectively. We also introduce the following discrete inner product and norm:

$$
\begin{aligned}
& (u, v)_{T, N}=\sum_{j=0}^{N} u\left(t_{T, j}^{N}\right) v\left(t_{T, j}^{N}\right) \omega_{T, j}^{N}, \\
& \|v\|_{T, N}=(v, v)_{T, N}^{\frac{1}{2}} .
\end{aligned}
$$

According to (5), for any $\varphi \in \mathcal{P}_{N}(0, T)$ and $\phi \cdot \psi \in \mathcal{P}_{2 N}(0, T)$, one can obtain

$$
(\phi, \psi)_{T}=(\phi, \psi)_{T, N}, \quad\|\varphi\|_{T}=\|\varphi\|_{T, N} .
$$

For any $v \in \mathcal{C}[0, T)$, the shifted Legendre-Gauss interpolation $\mathcal{I}_{T, N} v(t) \in \mathcal{P}_{N}(0, T)$ is determined uniquely by

$$
\mathcal{I}_{T, N} v\left(t_{T, j}^{N}\right)=v\left(t_{T, j}^{N}\right), \quad 0 \leq j \leq N
$$

Let $r$ be a nonnegative integer, $H^{r}(0, T)$ be the usual Sobolev space as defined in [17], and denote the semi-norm by $|\cdot|_{r, T}$, where $|U|_{r, T}=\left(\int_{0}^{T}\left(\frac{d^{r} U}{d t^{r}}\right)^{2} d t\right)^{\frac{1}{2}}$ and $U \in H^{r}(0, T)$. We have the following estimates.

Lemma 1 (see [15]) For any $u \in H^{r}(0, T)$ with integer $1 \leq r \leq N+1$, we have

$$
\begin{aligned}
& \left\|\mathcal{I}_{T, N} u-u\right\|_{T} \leq c T^{r} N^{-r}|u|_{r, T}, \\
& \left\|\left(\mathcal{I}_{T, N} u-u\right)^{\prime}\right\|_{T} \leq c T^{r-1} N^{\frac{3}{2}-r}|u|_{r, T} .
\end{aligned}
$$

Lemma 2 Let $u \in \mathcal{P}_{N+1}$, then

$$
\|u\|_{T, N}^{2} \leq 5\|u\|_{T}^{2}
$$

Proof Let $u \in \mathcal{P}_{N+1}, u(t)=\sum_{k=0}^{N+1} \tilde{u}_{T, k} L_{T, k}$, we have $\|u\|_{T}^{2}=\sum_{k=0}^{N+1} \tilde{u}_{T, k}^{2} \frac{T}{2 k+1}$. Moreover, according to (5) and (6), we can obtain

$$
\begin{aligned}
\|u\|_{T, N}^{2}= & \left(\sum_{k=0}^{N+1} \tilde{u}_{T, k} L_{T, k}, \sum_{k=0}^{N+1} \tilde{u}_{T, k} L_{T, k}\right)_{T, N} \\
= & \sum_{k=0}^{N} \tilde{u}_{T}^{2}\left(L_{T, k}, L_{T, k}\right)_{T}+2 \tilde{u}_{T, N} \tilde{u}_{T, N+1}\left(L_{T, N}, L_{T, N+1}\right)_{T, N} \\
& +\tilde{u}_{T, N+1}^{2}\left(L_{T, N+1}, L_{T, N+1}\right)_{T, N} .
\end{aligned}
$$


Due to (4), we deduce that

$$
\begin{aligned}
\left(L_{T, N+1}, L_{T, N+1}\right)_{T, N} \leq & \frac{T}{2}\left|\sum_{j=0}^{N} L_{N}^{2}\left(t_{j}^{N}\right) \omega_{j}^{N}\right| \leq \frac{T}{N+1}, \\
\left(L_{T, N}, L_{T, N+1}\right)_{T, N} \leq & \left|\sum_{j=0}^{N} L_{T, N}\left(t_{T, j}^{N}\right) L_{T, N+1}\left(t_{T, j}^{N}\right) \omega_{T, j}^{N}\right| \\
= & \frac{T}{2}\left|\sum_{j=0}^{N} L_{N}\left(t_{j}^{N}\right) L_{N+1}\left(t_{j}^{N}\right) \omega_{j}\right| \\
= & \frac{T}{2} \mid \frac{2}{(N+1)^{2}} L_{N}\left(t_{0}^{N}\right) L_{N+1}\left(t_{0}^{N}\right) \\
& +\sum_{j=1}^{N} \frac{-L_{N}^{2}\left(t_{j}^{N}\right)\left(1-t_{j}^{N}\right)}{(N+1)^{2}\left(L_{N}\left(t_{j}^{N}\right)\right)^{2}} \mid \leq \frac{T}{N+1} .
\end{aligned}
$$

Therefore, we have

$$
\begin{aligned}
\|u\|_{T, N}^{2} & \leq \sum_{k=0}^{N} \tilde{u}_{T, k}^{2} \frac{T}{2 k+1}+\left(\tilde{u}_{T, N}^{2}+\tilde{u}_{T, N+1}^{2}\right) \frac{T}{N+1}+\tilde{u}_{T, N+1}^{2} \frac{T}{N+1} \\
& \leq \sum_{k=0}^{N-1} \tilde{u}_{T, k}^{2} \frac{T}{2 k+1}+\tilde{u}_{T, N}^{2}\left(\frac{T}{2 N+1}+\frac{T}{N+1}\right)+\tilde{u}_{T, N+1}^{2} \frac{T}{N+1} \\
& \leq 5 \sum_{k=0}^{N+1} \tilde{u}_{T, k}^{2} \frac{T}{2 k+1}=5\|u\|_{T}^{2} .
\end{aligned}
$$

This completes the proof.

\subsection{The single-step scheme}

Now, we present the single-step scheme for the NDDEs (1). Denote the grid set by $\Lambda_{N}=$ $\left\{t_{T, k}^{N}: 0 \leq k \leq N\right\} \subset[0, T)$. The single-step Legendre-Gauss collocation approximation to (1) is to find $u^{N}(t) \in \mathcal{P}_{N+1}(0, T)$, such that

$$
\left\{\begin{array}{l}
\frac{d}{d t} u^{N}(t)=f\left(t, u^{N}(t), w(t), w^{\prime}(t)\right), \quad \forall t \in \Lambda_{N}, \\
u^{N}(0)=U(0)=V(0),
\end{array}\right.
$$

where

$$
w(t)=\left\{\begin{array}{ll}
u^{N}(\theta(t)), & \theta(t) \geq 0, \\
V(\theta(t)), & \theta(t)<0,
\end{array} \quad w^{\prime}(t)= \begin{cases}\frac{d}{d t} u^{N}(\theta(t)), & \theta(t) \geq 0, \\
V^{\prime}(\theta(t)), & \theta(t)<0 .\end{cases}\right.
$$

Here, we recall that $\theta(t)$ and $V(\cdot)$ are known functions. Denote

$$
\Lambda_{N}^{0}=\left\{t \in \Lambda_{N}: \theta(t)<0\right\}, \quad \Lambda_{N}^{1}=\left\{t \in \Lambda_{N}: \theta(t) \geq 0\right\} .
$$

It is an important problem how to resolve (10). Indeed, it is often used to resolve the discrete system (10) based on the Lagrange interpolation. However, it is well known that a 
Lagrange interpolation is not stable for large $N$. Hence, we propose a more stable approach by expanding $u^{N}(t)$ directly in terms of the shifted Legendre polynomials and solving the unknown coefficients of the collocation scheme (10). This approach is stable for large $N$, and much easier to implement.

To describe the numerical implementation of the collocation scheme (10), we expand the collocation solution as

$$
u^{N}(t)=\sum_{l=0}^{N+1} \hat{u}_{T, l}^{N} L_{T, l}(t) \in \mathcal{P}_{N+1}(0, T), \quad 0<t \leq T .
$$

Let $[l]$ be the integer part of $l$. According to [12], we have $\frac{d}{d t} L_{T, l}(t)=\frac{2}{T} \sum_{m=0}^{\left[\frac{l-1}{2}\right]}(2 l-4 m-$ 1) $L_{T, l-2 m-1}(t)$ and $L_{T, l}(0)=(-1)^{l}$. Hence, $(10)$ is equivalent to

$$
\left\{\begin{array}{l}
\sum_{l=1}^{N+1} a_{T, k, l}^{N} \hat{u}_{T, l}^{N}=f_{T, k}^{N}, \quad 0 \leq k \leq N \\
\sum_{l=0}^{N+1}(-1)^{l} \hat{u}_{T, l}^{N}=V(0)
\end{array}\right.
$$

where $a_{T, k, l}^{N}=\frac{2}{T} \sum_{m=0}^{\left[\frac{l-1}{2}\right]}(2 l-4 m-1) L_{T, l-2 m-1}\left(t_{T, k}^{N}\right), 0 \leq k \leq N, 1 \leq l \leq N+1$, and

$$
f_{T, k}^{N}=\left\{\begin{array}{cl}
f\left(t_{T, k}^{N}, \sum_{l=0}^{N+1} \hat{u}_{T, l}^{N} L_{T, l}\left(t_{T, k}^{N}\right), \sum_{l=0}^{N+1} \hat{u}_{T, l}^{N} L_{T, l}\left(\theta\left(t_{T, k}^{N}\right)\right),\right. & \\
\left.\left.\frac{d}{d t} \sum_{l=0}^{N+1} \hat{u}_{T, l}^{N} L_{T, l}(\theta(t))\right|_{t=t_{T, k}^{N}}\right), & t_{T, k}^{N} \in \Lambda_{N}^{1}, \\
f\left(t_{T, k}^{N}, \sum_{l=0}^{N+1} \hat{u}_{T, l}^{N} L_{T, l}\left(t_{T, k}^{N}\right), V\left(\theta\left(t_{T, k}^{N}\right)\right), V^{\prime}\left(\theta\left(t_{T, k}^{N}\right)\right)\right), & t_{T, k}^{N} \in \Lambda_{N}^{0} .
\end{array}\right.
$$

Let $A_{T}^{N}$ be the matrix with the entries $a_{T, k, l}^{N}, 0 \leq k \leq N, 1 \leq l \leq N+1$. We can give the matrix form for (12) as follows:

$$
\left\{\begin{array}{l}
A_{T}^{N} \hat{u}_{T}^{N}=F_{T}^{N}\left(\hat{u}_{T}^{N}\right) \\
\hat{u}_{T, 0}^{N}=V(0)-\sum_{l=1}^{N+1}(-1)^{l} \hat{u}_{T, l}^{N}
\end{array}\right.
$$

where $\hat{u}_{T}^{N}=\left(\hat{u}_{T, 1}^{N}, \hat{u}_{T, 2}^{N}, \ldots, \hat{u}_{T, N+1}^{N}\right)^{\prime}, F_{T}^{N}\left(\hat{u}_{T}^{N}\right)=\left(f_{T, 0}^{N}, f_{T, 1}^{N}, \ldots, f_{T, N}^{N}\right)^{\prime}$. In fact, (13) is a set of linear equations about $\left\{\hat{u}_{T, l}^{N}\right\}_{l=0}^{N+1}$. Therefore, we can solve (13) and recover the collocation solution $u^{N}(t), 0<t \leq T$ from (11).

\subsection{Error analysis}

In this subsection, we shall analyze the convergence of the scheme (10) with three assumptions of delay function $\theta(t)$, respectively. In particular, we prove the spectral accuracy of the numerical solution $u^{N}(t)$. Let $\mathcal{I}_{T, N}$ be the Legendre-Gauss interpolation operator as defined before. Let $E^{N}(t)=u^{N}(t)-\mathcal{I}_{T, N} U(t)$ and $G_{T, 2}^{N}(t)=\mathcal{I}_{T, N} \frac{d}{d t} U(t)-\frac{d}{d t} \mathcal{I}_{T, N} U(t)$. According to (1), we have

$$
\frac{d}{d t} \mathcal{I}_{T, N} U(t)=f\left(t, U(t), W(t), W^{\prime}(t)\right)-G_{T, 2}^{N}(t), \quad t \in \Lambda_{N} .
$$

Subtracting (14) from (10), we obtain

$$
\left\{\begin{array}{l}
\frac{d}{d t} E^{N}(t)=G_{T, 1}^{N}(t)+G_{T, 2}^{N}(t), \quad t \in \Lambda_{N} \\
E^{N}(0)=U(0)-\mathcal{I}_{T, N} U(0)
\end{array}\right.
$$

where $G_{T, 1}^{N}(t)=f\left(t, u^{N}(t), w(t), w^{\prime}(t)\right)-f\left(t, \mathcal{I}_{T, N} U(t), \mathcal{I}_{T, N} W(t), \mathcal{I}_{T, N} W^{\prime}(t)\right)$. 
Lemma 3 Let $U$ and $u^{N}$ be the solutions of (1) and (10), respectively, $U \in H^{r}(0, T)$ with integer $2 \leq r \leq N+1$, then

$$
\frac{1}{2}\left\|t^{-1}\left(E^{N}-E^{N}(0)\right)\right\|_{T}^{2}+T^{-1}\left|E^{N}(T)-E^{N}(0)\right|^{2} \leq 2\left\|\frac{d}{d t} E^{N}(t)\right\|_{T, N}^{2} .
$$

Proof Due to the proof of Lemma 1 in [14], we have

$$
\begin{aligned}
& 2\left(E^{N}-E^{N}(0), \frac{d}{d t}\left(t^{-1}\left(E^{N}-E^{N}(0)\right)\right)\right)_{T, N} \\
& \quad=-\left\|t^{-1}\left(E^{N}-E^{N}(0)\right)\right\|_{T}^{2}+T^{-1}\left|E^{N}(T)-E^{N}(0)\right|^{2} .
\end{aligned}
$$

On the other hand, we derive that

$$
\frac{d}{d t}\left(t^{-1}\left(E^{N}(t)-E^{N}(0)\right)\right)=-t^{-2}\left(E^{N}(t)-E^{N}(0)\right)+t^{-1} \frac{d}{d t} E^{N}(t), \quad t \in \Lambda_{N} .
$$

Hence, by (5), we deduce that

$$
\begin{aligned}
& 2\left(E^{N}-E^{N}(0), \frac{d}{d t}\left(t^{-1}\left(E^{N}-E^{N}(0)\right)\right)\right)_{T, N} \\
& \quad \leq-2\left\|t^{-1}\left(E^{N}-E^{N}(0)\right)\right\|_{T}^{2}+\frac{1}{2}\left\|t^{-1}\left(E^{N}-E^{N}(0)\right)\right\|_{T}^{2}+2\left\|\frac{d}{d t} E^{N}\right\|_{T, N}^{2} .
\end{aligned}
$$

This completes the proof.

Now, we consider three cases according to the delay terms and analyze the numerical errors. Here, $\beta, \beta_{1}$, and $\beta_{2}$ denote any positive numbers less than $\frac{1}{4}$.

Case 2.1 Consider (10) with the following delay:

$$
\theta(t)=\lambda t, \quad 0<\lambda \leq 1
$$

In this situation, no values of $U(t)$ are needed in the delay term behind 0 , therefore, no discontinuities propagate from 0 . Then the solution is regular according to the regularity of $f$ and $\theta$.

Assume that $f(t, x, y, z)$ satisfies the following Lipschitz conditions in $x, y$, and $z$. That is, there exist real numbers $r_{1} \geq 0, r_{2} \geq 0$, and $0 \leq r_{3}<\frac{1}{\sqrt{ } \lambda}$ such that

$$
\begin{aligned}
& \left|f\left(t, x_{1}, y, z\right)-f\left(t, x_{2}, y, z\right)\right| \leq r_{1}\left|x_{1}-x_{2}\right| \\
& \left|f\left(t, x, y_{1}, z\right)-f\left(t, x, y_{2}, z\right)\right| \leq r_{2}\left|y_{1}-y_{2}\right| \\
& \left|f\left(t, x, y, z_{1}\right)-f\left(t, x, y, z_{2}\right)\right| \leq r_{3}\left|z_{1}-z_{2}\right| .
\end{aligned}
$$

Theorem 1 Consider the NDDE (1), where $f(t, x, y, z)$ is $C^{r}$-continuous in $[0, T] \times R^{d} \times$ $R^{d} \times R^{d}$, and the initial function $V(t)$ is $C^{r}$-continuous. If the conditions (17)-(20) hold, 
$U \in H^{r}(0, T)$ with $2 \leq r \leq N+1$, and, for certain $\delta, \delta_{0}, \delta_{1}, \hat{\delta}, \tilde{\delta}>0$,

$$
\begin{aligned}
& \frac{\left(1+\delta_{0}\right)\left(1+\delta^{-1}\right)\left(1+\delta_{1}\right) 5 T^{2} r_{1}^{2}+\left(1+\delta_{0}\right)\left(1+\delta^{-1}\right)\left(1+\delta_{1}^{-1}\right) 5 \lambda^{-1} T^{2} r_{2}^{2}}{1-\left(1+\delta_{0}\right)(1+\delta)(1+\hat{\delta})(1+\tilde{\delta}) \lambda r_{3}^{2}} \\
& \quad \leq \beta<\frac{1}{4},
\end{aligned}
$$

then we have

$$
\left\|U-u^{N}\right\|_{T}^{2} \leq c_{\beta} T^{2 r} N^{3-2 r}|U|_{r, T}^{2}, \quad\left|U(T)-u^{N}(T)\right|^{2} \leq c_{\beta} T^{2 r-1} N^{3-2 r}|U|_{r, T}^{2} .
$$

In particular, $\max _{t \in[0, T]}\left|U(t)-u^{N}(t)\right|^{2} \leq c_{\beta} T^{2 r-1} N^{3-2 r}|U|_{r, T}^{2}$, where $c_{\beta}$ is a positive constant depending only on $\beta$.

Proof Obviously, in this case, $\Lambda_{N}^{0}=\emptyset$. Denote

$$
\begin{aligned}
\left\|\tilde{G}_{T, 1}^{N}\right\|_{T, N}^{2}= & \left(1+\delta^{-1}\right) \| f\left(t, u^{N}(t), w(t), \mathcal{I}_{T, N} \frac{d}{d t} W(t)\right) \\
& -f\left(t, \mathcal{I}_{T, N} U(t), \mathcal{I}_{T, N} W(t), \mathcal{I}_{T, N} \frac{d}{d t} W(t)\right) \|_{T, N}^{2} .
\end{aligned}
$$

Together with (6)-(8) and (20), for any $\tilde{\delta}, \delta>0$, we can obtain

$$
\begin{aligned}
\left\|G_{T, 1}^{N}\right\|_{T, N}^{2} \leq & (1+\delta)(1+\tilde{\delta}) r_{3}^{2}\left\|\frac{d}{d t} w(t)-\frac{d}{d t} W(t)\right\|_{T}^{2} \\
& +(1+\delta)\left(1+\tilde{\delta}^{-1}\right) r_{3}^{2}\left\|\frac{d}{d t} W(t)-\mathcal{I}_{T, N} \frac{d}{d t} W(t)\right\|_{T}^{2}+\left\|\tilde{G}_{T, 1}^{N}\right\|_{T, N}^{2}
\end{aligned}
$$

where

$$
\begin{aligned}
\left\|\frac{d}{d t} w(t)-\frac{d}{d t} W(t)\right\|_{T}^{2} & =\int_{\theta(0)}^{\theta(T)} \lambda\left[\frac{d}{d t} u^{N}(t)-\frac{d}{d t} U(t)\right]^{2} d t \\
& \leq \lambda \int_{0}^{T}\left[\frac{d}{d t} u^{N}(t)-\frac{d}{d t} U(t)\right]^{2} d t=\lambda\left\|\frac{d}{d t}\left(u^{N}-U\right)\right\|_{T}^{2}
\end{aligned}
$$

and

$$
\begin{aligned}
\left\|\frac{d}{d t} W(t)-\mathcal{I}_{T, N} \frac{d}{d t} W(t)\right\|_{T}^{2} & \leq c T^{2(r-1)} N^{-2(r-1)}\left|U^{\prime}(\theta(t))\right|_{r-1, T}^{2} \\
& \leq c T^{2 r-2} N^{2-2 r}|U(t)|_{r, T}^{2} .
\end{aligned}
$$

Applying (8), we have

$$
\begin{aligned}
& \left\|G_{T, 1}^{N}\right\|_{T, N}^{2}-\left\|\tilde{G}_{T, 1}^{N}\right\|_{T, N}^{2} \\
& \quad \leq(1+\delta)(1+\tilde{\delta})(1+\hat{\delta}) r_{3}^{2} \lambda\left\|\frac{d}{d t}\left(u^{N}-\mathcal{I}_{T, N} U\right)\right\|_{T}^{2}
\end{aligned}
$$




$$
\begin{aligned}
& +(1+\delta)(1+\tilde{\delta})\left(1+\hat{\delta}^{-1}\right) r_{3}^{2} \lambda\left\|\frac{d}{d t}\left(\mathcal{I}_{T, N} U-U\right)\right\|_{T}^{2}+c T^{2 r-2} N^{2-2 r}|U|_{r, T}^{2} \\
\leq & (1+\delta)(1+\tilde{\delta})(1+\hat{\delta}) r_{3}^{2} \lambda\left\|\frac{d}{d t} E^{N}\right\|_{T}^{2}+c T^{2 r-2} N^{3-2 r}|U|_{r, T}^{2} .
\end{aligned}
$$

By (15), we have

$$
\begin{aligned}
\left\|\frac{d}{d t} E^{N}\right\|_{T, N}^{2} \leq & \left(1+\delta_{0}\right)\left\|G_{T, 1}^{N}\right\|_{T, N}^{2}+\left(1+\delta_{0}^{-1}\right)\left\|G_{T, 2}^{N}\right\|_{T, N}^{2} \\
\leq & (1+\delta)(1+\tilde{\delta})(1+\hat{\delta})\left(1+\delta_{0}\right) \lambda r_{3}^{2}\left\|\frac{d}{d t} E^{N}\right\|_{T, N}^{2} \\
& +c T^{2 r-2} N^{3-2 r}|U|_{r, T}^{2}+\left(1+\delta_{0}\right)\left\|\tilde{G}_{T, 1}^{N}\right\|_{T, N}^{2} \\
& +\left(1+\delta_{0}^{-1}\right)\left\|G_{T, 2}^{N}\right\|_{T, N}^{2},
\end{aligned}
$$

where

$$
\begin{aligned}
\left\|G_{T, 2}^{N}\right\|_{T, N}^{2} & =\left\|G_{T, 2}^{N}\right\|_{T}^{2} \\
& \leq(1+\delta)\left\|\frac{d}{d t}\left(\mathcal{I}_{T, N} U-U\right)\right\|_{T}^{2}+\left(1+\delta^{-1}\right)\left\|\mathcal{I}_{T, N} \frac{d}{d t} U-\frac{d}{d t} U\right\|_{T}^{2} \\
& \leq c T^{2 r-2} N^{3-2 r}|U|_{r, T}^{2} .
\end{aligned}
$$

The above fact leads to

$$
\left\|\frac{d}{d t} E^{N}\right\|_{T, N}^{2} \leq \frac{\left(1+\delta_{0}\right)}{\hat{c}}\left\|\tilde{G}_{T, 1}^{N}\right\|_{T, N}^{2}+c T^{2 r-2} N^{3-2 r}|U|_{r, T}^{2}
$$

where $\hat{c}=1-(1+\delta)(1+\tilde{\delta})(1+\hat{\delta})\left(1+\delta_{0}\right) \lambda r_{3}^{2}$.

Let us estimate $\left\|\tilde{G}_{T, 1}^{N}\right\|_{T, N}^{2}$. Clearly, by (9) and (18)-(19), we have

$$
\begin{aligned}
\left\|\tilde{G}_{T, 1}^{N}\right\|_{T, N}^{2} \leq & \left(1+\delta^{-1}\right)\left(1+\delta_{1}\right) r_{1}^{2}\left\|E^{N}\right\|_{T, N}^{2} \\
& +\left(1+\delta^{-1}\right)\left(1+\delta_{1}^{-1}\right) r_{2}^{2}\left\|w(t)-\mathcal{I}_{T, N} W(t)\right\|_{T, N}^{2} \\
\leq & \left(1+\delta^{-1}\right)\left(1+\delta_{1}\right) 5 r_{1}^{2}\left\|E^{N}\right\|_{T}^{2} \\
& +\left(1+\delta^{-1}\right)\left(1+\delta_{1}^{-1}\right) 5 r_{2}^{2}\left\|w(t)-\mathcal{I}_{T, N} W(t)\right\|_{T}^{2}
\end{aligned}
$$

where

$$
\begin{aligned}
& \left\|w(t)-\mathcal{I}_{T, N} W(t)\right\|_{T}^{2} \\
& \leq(1+\varepsilon)\|w(t)-W(t)\|_{T}^{2}+\left(1+\varepsilon^{-1}\right)\left\|W(t)-\mathcal{I}_{T, N} W(t)\right\|_{T}^{2} \\
& \leq(1+\varepsilon) \int_{0}^{T}\left[u^{N}(\theta(t))-U(\theta(t))\right]^{2} d t+c \varepsilon^{-1} T^{2 r} N^{-2 r}|U|_{r, T}^{2} \\
& \quad \leq(1+\varepsilon) \lambda^{-1}\left\|u^{N}-U\right\|_{T}^{2}+c \varepsilon^{-1} T^{2 r} N^{-2 r}|U|_{r, T}^{2} .
\end{aligned}
$$


Thus, we can obtain

$$
\begin{aligned}
\left\|\tilde{G}_{T, 1}^{N}\right\|_{T, N}^{2} \leq & \left(1+\delta^{-1}\right)\left(1+\delta_{1}\right) 5 r_{1}^{2}\left\|E^{N}\right\|_{T}^{2} \\
& +\left(1+\delta^{-1}\right)\left(1+\delta_{1}^{-1}\right)(1+\varepsilon) 5 \lambda^{-1} r_{2}^{2}\left\|u^{N}-U\right\|_{T}^{2} \\
& +c \varepsilon^{-1} T^{2 r-2} N^{3-2 r}|U|_{r, T}^{2}
\end{aligned}
$$

According to (16), we have

$$
\begin{aligned}
\frac{1}{2}\left\|t^{-1}\left(E^{N}-E^{N}(0)\right)\right\|_{T}^{2}+T^{-1}\left|E^{N}(T)-E^{N}(0)\right|^{2} \\
\leq \frac{2\left(1+\delta_{0}\right)\left(1+\delta^{-1}\right)\left(1+\delta_{1}\right) 5 r_{1}^{2}}{\hat{c}}\left\|E^{N}\right\|_{T}^{2} \\
\quad+\frac{2\left(1+\delta_{0}\right)\left(1+\delta^{-1}\right)\left(1+\delta_{1}^{-1}\right)(1+\varepsilon) 5 \lambda^{-1} r_{2}^{2}}{\hat{c}}\left\|u^{N}-U\right\|_{T}^{2} \\
\quad+c \varepsilon^{-1} T^{2 r} N^{3-2 r}|U|_{r, T}^{2} .
\end{aligned}
$$

Moreover, we can obtain

$$
\left\|E^{N}\right\|_{T}^{2} \leq(1+\varepsilon) T^{2}\left\|t^{-1}\left(E^{N}-E^{N}(0)\right)\right\|_{T}^{2}+\left(1+\varepsilon^{-1}\right)\left\|E^{N}(0)\right\|_{T}^{2}
$$

and

$$
\begin{aligned}
& \frac{1}{2}\left\|E^{N}\right\|_{T}^{2}+(1+\varepsilon) T\left|E^{N}(T)-E^{N}(0)\right|^{2} \\
& \leq(1+\varepsilon) T^{2}\left(\frac{1}{2}\left\|t^{-1}\left(E^{N}-E^{N}(0)\right)\right\|_{T}^{2}+T^{-1}\left|E^{N}(T)-E^{N}(0)\right|^{2}\right)+c \varepsilon^{-1} T\left(E^{N}(0)\right)^{2} \\
& \leq \frac{2(1+\varepsilon)\left(1+\delta_{0}\right)\left(1+\delta^{-1}\right)\left(1+\delta_{1}\right) 5 T^{2} r_{1}^{2}}{\hat{c}}\left\|E^{N}\right\|_{T}^{2} \\
&+\frac{2\left(1+\delta_{0}\right)\left(1+\delta^{-1}\right)\left(1+\delta_{1}^{-1}\right)(1+\varepsilon)^{2} 5 T^{2} \lambda^{-1} r_{2}^{2}}{\hat{c}}\left\|u^{N}-U\right\|_{T}^{2} \\
&+c \varepsilon^{-1} T^{2 r} N^{3-2 r}|U|_{r, T}^{2}+c \varepsilon^{-1} T\left(E^{N}(0)\right)^{2} .
\end{aligned}
$$

Thanks to (7), we have

$$
\begin{aligned}
\left\|U-u^{N}\right\|_{T}^{2} & \leq(1+\varepsilon)\left\|E^{N}\right\|_{T}^{2}+\left(1+\varepsilon^{-1}\right)\left\|U-\mathcal{I}_{T, N} U\right\|_{T}^{2} \\
& \leq(1+\varepsilon)\left\|E^{N}\right\|_{T}^{2}+c \varepsilon^{-1} T^{2 r} N^{-2 r}|U|_{r, T}^{2} .
\end{aligned}
$$

The above result yields

$$
\begin{aligned}
\left(\frac{1}{2}-\right. & \left.\frac{2(1+\varepsilon)\left(1+\delta_{0}\right)\left(1+\delta^{-1}\right)\left(1+\delta_{1}\right) 5 T^{2} r_{1}^{2}}{\hat{c}}\right)\left\|U-u^{N}\right\|_{T}^{2} \\
& +(1+\varepsilon)^{2} T\left|E^{N}(T)-E^{N}(0)\right|^{2} \\
\leq & \frac{2(1+\varepsilon)^{3}\left(1+\delta_{0}\right)\left(1+\delta^{-1}\right)\left(1+\delta_{1}^{-1}\right) 5 \lambda^{-1} T^{2} r_{2}^{2}}{\hat{c}}\left\|u^{N}-U\right\|_{T}^{2} \\
& +c \varepsilon^{-1} T^{2 r} N^{3-2 r}|U|_{r, T}^{2}+c \varepsilon^{-1} T\left(E^{N}(0)\right)^{2} .
\end{aligned}
$$

Due to $t_{T, 0}^{N}=0$, we have $E^{N}(0)=0$. 
Let $\varepsilon=\left(\frac{1}{4 \beta}\right)^{1 / 3}-1>0$, then $(1+\varepsilon)^{3} \beta=\frac{1}{4}$. Thus, we can obtain

$$
\begin{aligned}
\hat{q} & =\frac{\left(1+\delta_{0}\right)\left(1+\delta^{-1}\right) 5 T^{2}\left((1+\varepsilon)\left(1+\delta_{1}\right) r_{1}^{2}+(1+\varepsilon)^{3}\left(1+\delta_{1}^{-1}\right) \lambda^{-1} r_{2}^{2}\right)}{1-\left(1+\delta_{0}\right)(1+\delta)(1+\tilde{\delta})(1+\hat{\delta}) \lambda r_{3}^{2}} \\
& <(1+\varepsilon)^{3} \frac{\left(1+\delta_{0}\right)\left(1+\delta^{-1}\right) 5 T^{2}\left(\left(1+\delta_{1}\right) r_{1}^{2}+\left(1+\delta_{1}^{-1}\right) \lambda^{-1} r_{2}^{2}\right)}{1-\left(1+\delta_{0}\right)(1+\delta)(1+\tilde{\delta})(1+\hat{\delta}) \lambda r_{3}^{2}} \\
& \leq(1+\varepsilon)^{3} \beta=\frac{1}{4} .
\end{aligned}
$$

By a combination of the above estimates, we deduce that

$$
\begin{aligned}
& \left\|U-u^{N}\right\|_{T}^{2} \leq c_{\beta} T^{2 r} N^{3-2 r}|U|_{r, T}^{2}, \\
& \left(E^{N}(T)\right)^{2} \leq 2\left|E^{N}(T)-E^{N}(0)\right|^{2}+2\left|E^{N}(0)\right|^{2} \leq c_{\beta} T^{2 r-1} N^{3-2 r}|U|_{r, T}^{2},
\end{aligned}
$$

where $c_{\beta}=\frac{c \varepsilon^{-1}}{\frac{1}{2}-2 \hat{q}}$. Consequently, we have

$$
\begin{aligned}
\left|U(T)-u^{N}(T)\right|^{2} & \leq 2\left|\mathcal{I}_{T, N} U(T)-U(T)\right|^{2}+2\left|E^{N}(T)\right|^{2} \\
& \leq c_{\beta} T^{2 r-1} N^{3-2 r}|U|_{r, T}^{2}, \\
\left\|\frac{d}{d t}\left(U-u^{N}\right)\right\|_{T}^{2} & \leq 2\left\|\frac{d}{d t} E^{N}\right\|_{T}^{2}+2\left\|\frac{d}{d t}\left(U-\mathcal{I}_{T, N} U\right)\right\|_{T}^{2} \leq c_{\beta} T^{2 r-2} N^{3-2 r}|U|_{r, T}^{2} .
\end{aligned}
$$

From (3.9) of [13], it can be seen that $\max _{t \in[0, T]}|v(t)|^{2} \leq \frac{2}{T}\|v\|_{T}^{2}+2 T\left\|\frac{d v}{d t}\right\|_{T}^{2}$ for any $v \in$ $H^{1}(0, T)$. Hence, we deduce that $\max _{t \in[0, T]}\left|U(t)-u^{N}(t)\right|^{2} \leq c_{\beta} T^{2 r-1} N^{3-2 r}|U|_{r, T}^{2}$.

Remark 1 For (1) and (10) without the neutral term, the convergence results are the same as Case I in [14].

Remark 2 The condition (21) is necessary for the proof, but it is not sufficient. Some numerical examples do not satisfy this condition, but the numerical scheme still converges. This remark also applies to the multiple-domain case.

Case 2.2 Assume that the delay function $\theta(t)$ satisfies

$$
\theta(t)=t-\tau, \quad \tau>0, t \in[0, T]
$$

In this situation, the points $t_{k}=k \tau(k=0,1, \ldots)$ are primary discontinuity points. Therefore, there is no discontinuity point when $T \leq \tau$. Moreover, $f(t, x, y, z)$ satisfies the Lipschitz conditions (18)-(20), where $r_{3}$ satisfies $0 \leq r_{3}<1$.

Theorem 2 Consider the NDDE (1) with $T \leq \tau$, where $f(t, x, y, z)$ is $C^{r}$-continuous in $[0, T] \times R^{d} \times R^{d} \times R^{d}$, and the initial function $V(t)$ is $C^{r}$-continuous. If the conditions (18)-(20) and (22) hold, $U \in H^{r}(0, T)$ with integer $2 \leq r \leq N+1,(1+\delta) 5 T^{2} r_{1}^{2} \leq \beta_{1}<\frac{1}{4}$, and $\frac{5(T-\tau)^{2}\left(1+\delta_{0}\right)\left(1+\delta^{-1}\right)\left(\left(1+\delta_{1}\right) r_{1}^{2}+\left(1+\delta_{1}^{-1}\right) r_{2}^{2}\right)}{1-\left(1+\delta_{0}\right)(1+\delta)(1+\delta)(1+\tilde{\delta}) r_{3}^{2}} \leq \beta_{2}<\frac{1}{4}$ for certain $\delta, \delta_{0}, \delta_{1}, \hat{\delta}, \tilde{\delta}>0$, then we have

$$
\begin{aligned}
& \left\|U-u^{N}\right\|_{T}^{2} \leq c_{\beta_{1}, \beta_{2}} T^{2 r} N^{3-2 r}|U|_{r, T}^{2} \\
& \left|U(T)-u^{N}(T)\right|^{2} \leq c_{\beta_{1}, \beta_{2}} T^{2 r-1} N^{3-2 r}|U|_{r, T}^{2} .
\end{aligned}
$$


In particular, $\max _{t \in[0, T]}\left|U(t)-u^{N}(t)\right|^{2} \leq c_{\beta_{1}, \beta_{2}} T^{2 r-1} N^{3-2 r}|U|_{r, T}^{2}$, where $c_{\beta_{1}, \beta_{2}}$ is a positive constant depending only on $\beta_{1}, \beta_{2}$.

Proof As $\tau \geq T, \theta(t)<0$ for $t \in[0, T]$. In this case, $\Lambda_{N}^{1}=\emptyset$. Hence, by virtue of (18), we have $\left\|G_{T, 1}^{N}\right\|_{T, N}^{2} \leq r_{1}^{2}\left\|E^{N}\right\|_{T, N}^{2}$. Due to (9) and (16), we derive that

$$
\begin{aligned}
& \left(\frac{1}{2}-2(1+\varepsilon)(1+\delta) 5 T^{2} r_{1}^{2}\right)\left\|E^{N}\right\|_{T}^{2}+(1+\varepsilon) T\left|E^{N}(T)-E^{N}(0)\right|^{2} \\
& \quad \leq c \varepsilon^{-1} T^{2 r} N^{3-2 r}|U|_{r, T}^{2} .
\end{aligned}
$$

Let $\varepsilon=\left(\frac{1}{4 \beta_{1}}\right)>0$, then

$$
\hat{q}_{1}=(1+\varepsilon)(1+\delta) 5 T^{2} r_{1}^{2}<(1+\varepsilon) \beta_{1}=1 / 4 .
$$

Thus,

$$
\left\|E^{N}\right\|_{T}^{2} \leq c_{\beta_{1}} T^{2 r} N^{3-2 r}|U|_{r, T}^{2}
$$

and

$$
\begin{aligned}
&\left\|U-u^{N}\right\|_{T}^{2} \leq(1+\varepsilon)\left\|E^{N}\right\|_{T}^{2}+\left(1+\varepsilon^{-1}\right)\left\|U-\mathcal{I}_{T, N} U\right\|_{T}^{2} \\
& \leq c_{\beta_{1}} T^{2 r} N^{3-2 r}|U|_{r, T}^{2}, \\
&\left|U(T)-u^{N}(T)\right|^{2} \leq 2\left|\mathcal{I}_{T, N} U(T)-U(T)\right|^{2}+2\left|E^{N}(T)\right|^{2} \\
& \leq c_{\beta_{1}} T^{2 r-1} N^{3-2 r}|U|_{r, T}^{2}, \\
&\left\|\frac{d}{d t}\left(U-u^{N}\right)\right\|_{T}^{2} \leq 2\left\|\frac{d}{d t} E^{N}\right\|_{T}^{2}+2\left\|\frac{d}{d t}\left(U-\mathcal{I}_{T, N} U\right)\right\|_{T}^{2} \\
& \leq c_{\beta_{1}} T^{2 r-2} N^{3-2 r}|U|_{r, T}^{2}, \\
& \max _{t \in[0, T]}\left|U(t)-u^{N}(t)\right|^{2} \leq c_{\beta_{1}} T^{2 r-1} N^{3-2 r}|U|_{r, T}^{2},
\end{aligned}
$$

where $c_{\beta_{1}}=\frac{c \varepsilon^{-1}}{\frac{1}{2}-2 \hat{q}_{1}}$.

Case 2.3 Assume that the delay function $\theta(t)$ satisfies

$$
\left\{\begin{array}{l}
D_{1}: \theta(t)=t-\tau(t), \quad \tau \in C^{d}(I), d \geq 1 \\
D_{2}: \tau(t) \geq \tau_{0}>0, \quad t \in I \\
D_{3}: \theta \text { is strictly increasing on } I .
\end{array}\right.
$$

Now, we assume that $\left\{T_{k}\right\}_{k=0}^{\infty}$ are primary discontinuity points which satisfy $\theta\left(t_{k+1}\right)=t_{k}$, $t_{0}=0$. By the definition of $\theta(t)$, we conclude that $t_{k+1} \geq t_{k}+\tau_{0}, k=0,1, \ldots$ When $T \leq t_{0}$, there is no discontinuity point.

Moreover, the function $f(t, x, y, z)$ satisfies the Lipschitz conditions (18)-(20), but $r_{3}$ satisfies $0 \leq r_{3}<\frac{1}{\sqrt{c_{0}}}$, where $c_{0}=\max _{t \in[0, T]} \frac{1}{\left[\theta^{-1}(t)\right]^{\prime}}$. Due to (24), we see that the maximum and minimum of $\frac{1}{\left[\theta^{-1}(t)\right]^{\prime}}$ exist in $[0, T]$. 
Theorem 3 Consider the NDDE (1) with $T \leq t_{0}$ which satisfy $\theta\left(t_{0}\right)=0 . f(t, x, y, z)$ is $C^{r}$-continuous in $[0, T] \times R^{d} \times R^{d} \times R^{d}$, and the initial function $V(t)$ is $C^{r}$-continuous. If the conditions (18)-(20) and (24) hold, $U \in H^{r}(0, T)$ with integer $2 \leq r \leq N+1, c_{1}=$ $\min _{t \in[0, T]} \frac{1}{\left[\theta^{-1}(t)\right]^{2}},(1+\delta) 5 T^{2} r_{1}^{2} \leq \beta_{1}<\frac{1}{4}$ and

$$
\frac{\left(1+\delta_{0}\right)\left(1+\delta^{-1}\right)\left(1+\delta_{1}\right) 5 T^{2} r_{1}^{2}+\left(1+\delta_{0}\right)\left(1+\delta^{-1}\right)\left(1+\delta_{1}^{-1}\right) 5 c_{1} T^{2} r_{2}^{2}}{1-\left(1+\delta_{0}\right)(1+\delta)(1+\hat{\delta})(1+\tilde{\delta}) c_{0} r_{3}^{2}} \leq \beta_{2}<\frac{1}{4}
$$

for certain $\delta, \delta_{0}, \delta_{1}, \hat{\delta}, \tilde{\delta}>0$, then we have (23). In particular, $\max _{t \in[0, T]}\left|U(t)-u^{N}(t)\right|^{2} \leq$ $c_{\beta_{1}, \beta_{2}} T^{2 r-1} N^{3-2 r}|U|_{r, T}^{2}$, where $c_{\beta_{1}, \beta_{2}}$ is a positive constant depending only on $\beta_{1}, \beta_{2}$.

Proof Together with (24) and $\theta(0)<0$, we can find $t_{0}$ which satisfies $\theta\left(t_{0}\right)=0$. Let

$$
\left\{\begin{array}{l}
\Lambda_{N}^{0}=\left\{t_{T, k}^{N} \mid \theta\left(t_{T, k}^{N}\right)<0,0 \leq k \leq N\right\} \\
\Lambda_{N}^{1}=\left\{t_{T, k}^{N} \mid 0 \leq \theta\left(t_{T, k}^{N}\right) \leq T, 0 \leq k \leq N\right\}
\end{array}\right.
$$

If $t_{0} \geq T$, obviously, $\Lambda_{N}^{1}=\emptyset$. The proof is similar to $\tau \geq T$ in Case 2.2, thus we have

$$
\begin{aligned}
& \left\|U-u^{N}\right\|_{T}^{2} \leq c_{\beta_{1}} T^{2 r} N^{3-2 r}|U|_{r, T}^{2}, \quad\left|U(T)-u^{N}(T)\right|^{2} \leq c_{\beta_{1}} T^{2 r-1} N^{3-2 r}|U|_{r, T}^{2}, \\
& \max _{t \in[0, T]}\left|U(t)-u^{N}(t)\right|^{2} \leq c_{\beta_{1}} T^{2 r-1} N^{3-2 r}|U|_{r, T}^{2} .
\end{aligned}
$$

\subsection{Numerical results}

In this subsection, we give some numerical results to illustrate the efficiency of our singlestep algorithm.

Example 1 Consider the following equation:

$$
\left\{\begin{array}{l}
\frac{d}{d t} u(t)=\frac{1}{2} u(t)+\frac{1}{4} e^{\frac{t}{2}} u\left(\frac{t}{2}\right)+\frac{1}{4} e^{\frac{t}{2}} u^{\prime}\left(\frac{t}{2}\right), \quad 0 \leq t \leq T, \\
u(0)=1
\end{array}\right.
$$

The exact solution is $u(t)=e^{t}$. Obviously, the conditions (18)-(20) hold with $r_{1}=\frac{1}{2}, r_{2}=$ $\frac{1}{4} e^{\frac{T}{2}}$, and $r_{3}=\frac{1}{4} e^{\frac{T}{2}}$.

Moreover, the inequality (21) is satisfied for $T=0.3$. But it is no longer valid for $T=1$. In Figure 1, we plot the numerical errors at $t=T$ for $T=0.3$ and $T=1$, respectively. It indicates that the numerical errors decay exponentially as $N$ increases. In particular, we can observe that our algorithm is still valid even if the condition (21) is not satisfied.

Example 2 Consider the following equation:

$$
\left\{\begin{array}{l}
\frac{d}{d t} u(t)=\frac{1}{2} u(t)+\frac{1}{4} e^{\frac{1}{2}} u\left(t-\frac{1}{2}\right)+\frac{1}{4} e^{\frac{1}{2}} u^{\prime}\left(t-\frac{1}{2}\right), \quad 0 \leq t \leq T, \\
u(t)=e^{t}, \quad t<0 \\
u(0)=1
\end{array}\right.
$$

The exact solution is $u(t)=e^{t}$. Obviously, the conditions (18)-(20) hold with $r_{1}=\frac{1}{2}, r_{2}=$ $\frac{1}{4} e^{\frac{1}{2}}$, and $r_{3}=\frac{1}{4} e^{\frac{1}{2}}$. 


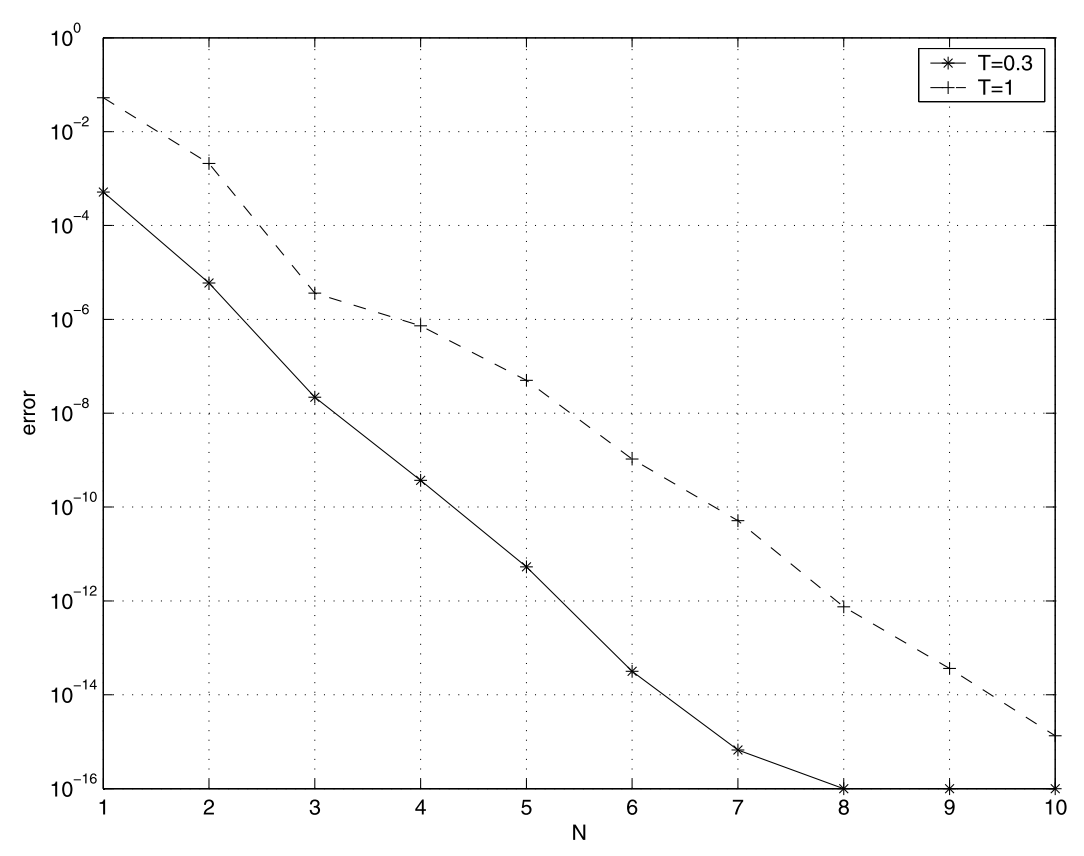

Figure 1 The numerical errors of (25) at $t=T$ for $T=0.3$ and $T=1$.

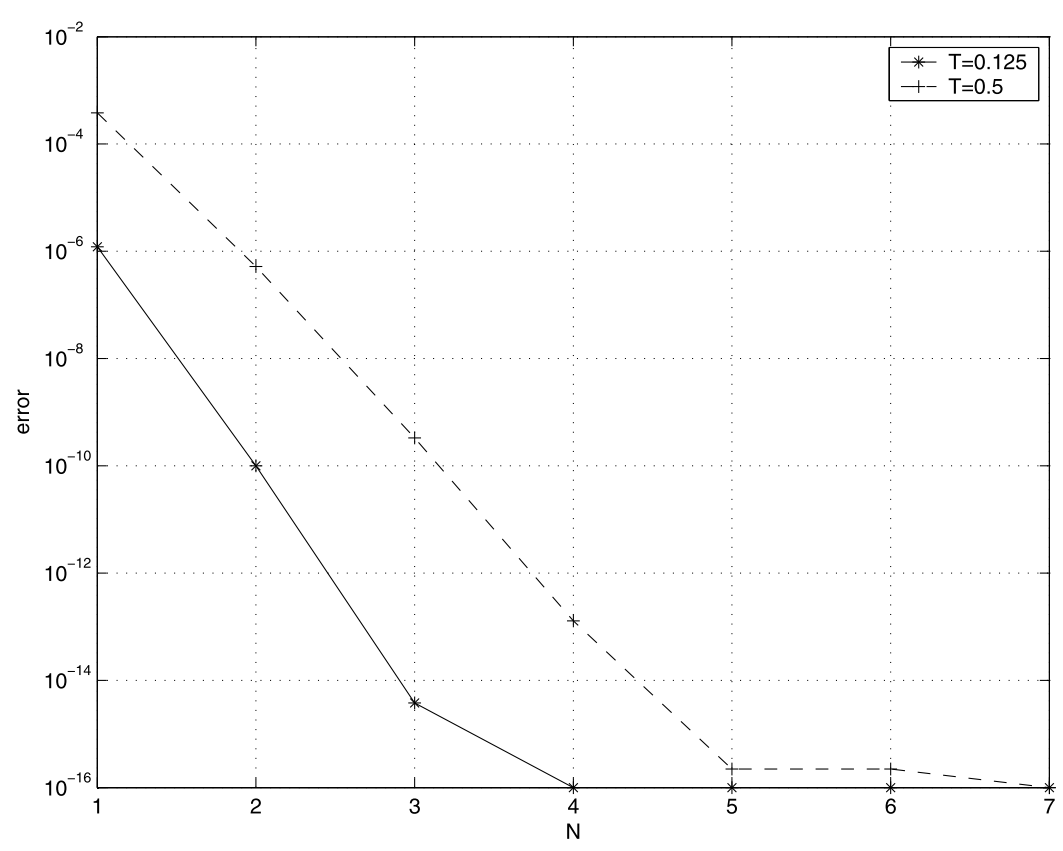

Figure 2 The numerical errors of (26) or (27) at $t=T$ for $T=0.125$ and $T=0.5$.

Moreover, the inequality $5 T^{2} r_{1}^{2} \leq \beta_{1}<\frac{1}{4}$ in Theorem 2 is satisfied for $T=0.125$. But it is no longer valid for $T=0.5$. In Figure 2, we plot the numerical errors at $t=T$ for $T=0.125$ and $T=0.5$, respectively. It indicates that the numerical errors decay exponentially as $N$ increases. In particular, we can observe that our algorithm is still valid even if the above inequality is not satisfied. 
Example 3 Consider the following equation:

$$
\left\{\begin{array}{l}
\frac{d}{d t} u(t)=\frac{1}{2} u(t)+\frac{1}{4} e^{\frac{t+1}{2}} u(\theta(t))+\frac{1}{4} e^{\frac{t+1}{2}} u^{\prime}(\theta(t)), \quad 0 \leq t \leq T \\
u(t)=e^{t}, \quad t<0 \\
u(0)=1
\end{array}\right.
$$

where $\theta(t)=t-\left(\frac{t}{2}+\frac{1}{2}\right)$. The exact solution is $u(t)=e^{t}$. Obviously, the conditions (18)-(20) hold with $r_{1}=\frac{1}{2}, r_{2}=\frac{1}{4} e^{\frac{T+1}{2}}$, and $r_{3}=\frac{1}{4} e^{\frac{T+1}{2}}$. The discrete scheme of (27) is the same as (26). So, we can also see the convergence of numerical solution of this equation in Figure 2. Here, the inequality $5 T^{2} r_{1}^{2} \leq \beta_{1}<\frac{1}{4}$ in Theorem 3 is satisfied for $T=0.125$. But it is no longer valid for $T=0.5$. In Figure 2, we plot the numerical errors at $t=T$ for $T=0.125$ and $T=0.5$, respectively. It indicates that the numerical errors decay exponentially as $N$ increases. In particular, we can observe that our algorithm is still valid even if the above inequality is not satisfied.

\section{The multiple-domain Legendre-Gauss collocation method}

We investigate the single-step Legendre-Gauss collocation method in Section 2. The numerical errors decay very rapidly as $N$ and $r$ increase. While the single-step collocation method provides accurate results, it is not suitable for resolving the discrete system (13) with large $T$. When there are some discontinuity points on $[0, T]$, the single-step method cannot be employed. We shall partition the interval $[0, T]$ into a finite number of subintervals by a set of points $\left\{T_{k}\right\}_{k=0}^{M}$. The primary discontinuity points on $[0, T]$ should be all contained in the set of $\left\{T_{k}\right\}_{k=0}^{M}$ such that the solution $U(t)$ is continuous in the interior of each subinterval. Then we solve the equations subsequently on each subinterval.

\subsection{The multiple-domain scheme}

Now, we describe the multiple-domain scheme. Let $M$ and $N_{m}, 1 \leq m \leq M$ be any positive integers. Decompose the interval $[0, T]$ into $M$ subintervals $\left[T_{m-1}, T_{m}\right], 1 \leq m \leq M$, such that the set of $T_{m}$ includes all breaking points, where $T_{0}=0$ and $T_{M}=T$. Let $\tau_{m}=T_{m}-$ $T_{m-1}, 1 \leq m \leq M$. We shall use $u_{m}^{N_{m}}(t) \in \mathcal{P}_{N_{m+1}}\left(0, \tau_{m}\right)$ to approximate the solution $U$ in the subinterval $\left[T_{m-1}, T_{m}\right]$.

Firstly, replacing $T$ and $N$ by $\tau_{1}$ and $N_{1}$ in (10) and all other formulas in Section 2.2, we can derive an alternative algorithm, with which we obtain the numerical solution $u_{1}^{N_{1}} \in$ $\mathcal{P}_{N_{1}+1}\left(0, \tau_{1}\right)$. Then we evaluate the numerical solutions $u_{m}^{N_{m}} \in \mathcal{P}_{N_{m}+1}\left(0, \tau_{m}\right), 2 \leq m \leq M$, step by step. Finally, the global numerical solution of (1) is given by

$$
u^{N}\left(T_{m-1}+t\right)=u_{m}^{N_{m}}(t), \quad 0 \leq t \leq \tau_{m}, 1 \leq m \leq M .
$$

We present the numerical scheme for $u_{m}^{N_{m}}(t)$. Denote the nodes and the corresponding Christoffel numbers of the shifted Legendre-Gauss interpolation on the interval $\left(0, \tau_{m}\right)$ by $t_{\tau_{m}, k}^{N_{m}}$ and $\omega_{\tau_{m}, k}^{N_{m}}, 0 \leq k \leq N_{m}$, respectively.

Let

$$
\left\{\begin{array}{l}
\Lambda_{N, m}^{0}=\left\{t_{\tau_{m}, k}^{N_{m}} \mid \theta\left(T_{m-1}+t_{\tau_{m, k}, N_{m}}^{N_{m}}\right)<0,0 \leq k \leq N_{m}\right\}, \\
\Lambda_{N, m}^{j}=\left\{t_{\tau_{m}, k}^{N_{m}} \mid \theta\left(T_{m-1}+t_{\tau_{m}, k}^{N_{m}}\right) \in\left[T_{j-1}, T_{j}\right), 0 \leq k \leq N_{m}\right\}, \quad 1 \leq j \leq m .
\end{array}\right.
$$


The multiple-domain collocation method for (1) is to seek $u_{m}^{N_{m}}(t) \in \mathcal{P}_{N_{m}+1}\left(0, \tau_{m}\right)$, such that

$$
\left\{\begin{array}{l}
\frac{d}{d t} u_{m}^{N_{m}}(t)=f\left(T_{m-1}+t, u_{m}^{N_{m}}(t), w_{m}(t), w_{m}^{\prime}(t)\right), \quad t \in \Lambda_{N, m}^{j}, j \geq 0, \\
u_{m}^{N_{m}}(0)=u_{m-1}^{N_{m-1}}\left(\tau_{m-1}\right), \quad 2 \leq m \leq M,
\end{array}\right.
$$

where

$$
\begin{aligned}
w_{m}(t) & =u^{N}\left(\theta\left(T_{m-1}+t\right)\right) \\
& = \begin{cases}u_{m}^{N_{m}}\left(\theta\left(T_{m-1}+t\right)-T_{m-1}\right), & \theta\left(T_{m-1}+t\right) \in\left[T_{m-1}, T_{m}\right), \\
\ldots, & \\
u_{j}^{N_{j}}\left(\theta\left(T_{m-1}+t\right)-T_{j-1}\right), & \theta\left(T_{m-1}+t\right) \in\left[T_{j-1}, T_{j}\right), \\
\ldots, & \theta\left(T_{m-1}+t\right) \in\left[T_{0}, T_{1}\right), \\
u_{1}^{N_{1}}\left(\theta\left(T_{m-1}+t\right)-T_{0}\right), & \theta\left(T_{m-1}+t\right)<0 . \\
V\left(\theta\left(T_{m-1}+t\right)\right), & \end{cases}
\end{aligned}
$$

Denote $U_{m}(t)=U\left(T_{m-1}+t\right)$ for $0 \leq t \leq \tau_{m}$. By (1), we have

$$
\left\{\begin{array}{l}
\frac{d}{d t} U_{m}(t)=f\left(T_{m-1}+t, U_{m}(t), W_{m}(t), W_{m}^{\prime}(t)\right), \quad t \in \Lambda_{N, m}^{j}, j \geq 0 \\
U_{m}(0)=U_{m-1}\left(\tau_{m-1}\right), \quad 2 \leq m \leq M \\
U_{1}(0)=U(0)=V(0)
\end{array}\right.
$$

where

$$
\begin{aligned}
W_{m}(t) & =U\left(\theta\left(T_{m-1}+t\right)\right) \\
& = \begin{cases}U_{m}\left(\theta\left(T_{m-1}+t\right)-T_{m-1}\right), & \theta\left(T_{m-1}+t\right) \in\left[T_{m-1}, T_{m}\right), \\
\ldots, & \theta\left(T_{m-1}+t\right) \in\left[T_{j-1}, T_{j}\right), \\
U_{j}\left(\theta\left(T_{m-1}+t\right)-T_{j-1}\right), & \\
\ldots, & \theta\left(T_{m-1}+t\right) \in\left[T_{0}, T_{1}\right), \\
U_{1}\left(\theta\left(T_{m-1}+t\right)-T_{0}\right), & \theta\left(T_{m-1}+t\right)<0 . \\
V\left(\theta\left(T_{m-1}+t\right)\right), & \end{cases}
\end{aligned}
$$

It can be seen from (29) and (30) that the local numerical solution $u_{m}^{N_{m}}(t)$ is actually an approximation to the local exact solution $U_{m}(t)$, with the approximate initial data $u_{m}^{N_{m}}(0)=$ $u_{m-1}^{N_{m-1}}\left(\tau_{m-1}\right)$.

\subsection{Error analysis}

We now analyze the numerical errors. Let $E_{m}^{N_{m}}(t)=u_{m}^{N_{m}}(t)-\mathcal{I}_{\tau_{m}, N_{m}} U_{m}(t)$. Together with (29) and (30), we have

$$
\left\{\begin{array}{l}
\frac{d}{d t} E_{m}^{N_{m}}(t)=G_{\tau_{m}, 1}^{N_{m}}(t)+G_{\tau_{m}, 2}^{N_{m}}(t), \quad t \in \Lambda_{N, m}^{j}, j \geq 0, \\
E_{m}^{N_{m}}(0)=u_{m}^{N_{m}}(0)-\mathcal{I}_{\tau_{m}, N_{m}} U_{m}(0), \quad 2 \leq m \leq M, \\
E_{1}^{N_{1}}(0)=U(0)-\mathcal{I}_{\tau_{1}, N_{1}} U(0),
\end{array}\right.
$$

where

$$
G_{\tau_{m}, 1}^{N_{m}}(t)=f\left(T_{m-1}+t, u_{m}^{N_{m}}, w_{m}(t), w_{m}^{\prime}(t)\right)-f\left(T_{m-1}+t, \mathcal{I}_{\tau_{m}, N_{m}} U_{m}(t), W_{m}(t), W_{m}^{\prime}(t)\right),
$$

and $G_{\tau_{m}, 2}^{N_{m}}(t)=\mathcal{I}_{\tau_{m}, N_{m}} \frac{d}{d t} U_{m}(t)-\frac{d}{d t} \mathcal{I}_{\tau_{m}, N_{m}} U_{m}(t)$ for $t \in \Lambda_{N, m}^{j}$ and $j \geq 0$. 
Lemma 4 Let $u_{m}^{N_{m}}(t)$ and $U_{m}$ be the solutions of (29) and (30), respectively. If $U_{m} \in$ $H^{r}\left(0, \tau_{m}\right)$ with integer $2 \leq r \leq N_{m}+1$, then

$$
\frac{1}{2}\left\|t^{-1}\left(E_{m}^{N_{m}}-E_{m}^{N_{m}}(0)\right)\right\|_{\tau_{m}}^{2}+\tau_{m}^{-1}\left|E_{m}^{N_{m}}\left(\tau_{m}\right)-E_{m}^{N_{m}}(0)\right|^{2} \leq 2\left\|\frac{d}{d t} E_{m}^{N_{m}}\right\|_{\tau_{m}, N_{m}}^{2} .
$$

Proof Applying Lemma 3, we have

$$
\frac{d}{d t}\left(t^{-1}\left(E_{m}^{N_{m}}(t)-E_{m}^{N_{m}}(0)\right)\right)=-t^{-2}\left(E_{m}^{N_{m}}(t)-E_{m}^{N_{m}}(0)\right)+t^{-1} \frac{d}{d t} E_{m}^{N_{m}}(t)
$$

and

$$
\begin{aligned}
& 2\left(E_{m}^{N_{m}}-E_{m}^{N_{m}}(0), \frac{d}{d t}\left(t^{-1}\left(E_{m}^{N_{m}}-E_{m}^{N_{m}}(0)\right)\right)\right)_{\tau_{m}, N_{m}} \\
& \quad=-\left\|t^{-1}\left(E_{m}^{N_{m}}-E_{m}^{N_{m}}(0)\right)\right\|_{\tau_{m}}^{2}+\tau_{m}^{-1}\left|E_{m}^{N_{m}}\left(\tau_{m}\right)-E_{m}^{N_{m}}(0)\right|^{2},
\end{aligned}
$$

where $t \in \Lambda_{N, m}^{j}$ for $j \geq 0$. By (6) with $\tau_{m}$ and $N_{m}$ instead of $T$ and $N$, we deduce that

$$
\begin{aligned}
2( & \left.E_{m}^{N_{m}}-E_{m}^{N_{m}}(0), \frac{d}{d t}\left(t^{-1}\left(E_{m}^{N_{m}}-E_{m}^{N_{m}}(0)\right)\right)\right)_{\tau_{m}, N_{m}} \\
= & -2\left(E_{m}^{N_{m}}-E_{m}^{N_{m}}(0), t^{-2}\left(E_{m}^{N_{m}}-E_{m}^{N_{m}}(0)\right)\right)_{\tau_{m}} \\
& +2\left(t^{-1}\left(E_{m}^{N_{m}}-E_{m}^{N_{m}}(0)\right), \frac{d}{d t} E_{m}^{N_{m}}(t)\right)_{\tau_{m}, N_{m}} \\
\leq & -2\left\|t^{-1}\left(E_{m}^{N_{m}}-E_{m}^{N_{m}}(0)\right)\right\|_{\tau_{m}}^{2} \\
& +\frac{1}{2}\left\|t^{-1}\left(E_{m}^{N_{m}}-E_{m}^{N_{m}}(0)\right)\right\|_{\tau_{m}}^{2}+2\left\|\frac{d}{d t} E_{m}^{N_{m}}\right\|_{\tau_{m}, N_{m}}^{2} .
\end{aligned}
$$

This completes the proof.

We shall analyze the numerical errors in the following three cases. Let $\hat{\tau}=\max _{1 \leq j \leq M} \tau_{j}$ and $N=\min _{1 \leq j \leq M} N_{j}$. Assume that, for any $1 \leq i \leq j \leq M, \tau_{i} / \tau_{j}$ is bounded.

Case 3.1 Consider the delay function $\theta(t)$ satisfying the condition (17). The solution $U(t)$ is regular according to the regularity of $f$ and $\theta$. Assume that $f(t, x, y, z)$ satisfies the Lipschitz conditions (18)-(20), where $r_{1} \geq 0, r_{2} \geq 0$, and $0 \leq r_{3}<\frac{1}{\sqrt{\lambda}}$.

Theorem 4 Consider the NDDE (1), where $f(t, x, y, z)$ is $C^{r}$-continuous in $[0, T] \times R^{d} \times$ $R^{d} \times R^{d}$, and the initial function $V(t)$ is $C^{r}$-continuous. If the conditions (17)-(20) hold, $U \in H^{r}(0, T)$ with $2 \leq r \leq N+1$, and, for certain $\delta, \delta_{0}, \delta_{1}, \hat{\delta}, \tilde{\delta}>0$,

$$
\begin{aligned}
& \frac{\left(1+\delta_{0}\right)\left(1+\delta^{-1}\right)\left(1+\delta_{1}\right) 5 \tau_{m}^{2} r_{1}^{2}+\left(1+\delta_{0}\right)\left(1+\delta^{-1}\right)\left(1+\delta_{1}^{-1}\right) 5 \lambda^{-1} \tau_{m}^{2} r_{2}^{2}}{1-\left(1+\delta_{0}\right)(1+\delta)(1+\hat{\delta})(1+\tilde{\delta}) \lambda r_{3}^{2}} \\
& \quad \leq \beta<\frac{1}{4},
\end{aligned}
$$


then, for any $1 \leq m \leq M$, we have

$$
\begin{aligned}
& \left\|U-u^{N}\right\|_{L^{2}\left(T_{m-1}, T_{m}\right)}^{2} \leq c_{\beta} \hat{\tau}^{2 r} N^{3-2 r}|U|_{r, T_{m}}^{2} \\
& \left|U\left(T_{m}\right)-u^{N}\left(T_{m}\right)\right|^{2} \leq c_{\beta} \hat{\tau}^{2 r-1} N^{3-2 r}|U|_{r, T_{m}}^{2}
\end{aligned}
$$

In particular, $\max _{t \in\left[T_{m-1}, T_{m}\right]}\left|U(t)-u^{N}(t)\right|^{2} \leq c_{\beta} \hat{\tau}^{2 r} N^{3-2 r}|U|_{r, T_{m}}^{2}$, where $c_{\beta}$ is a positive constant depending only on $\beta$.

Proof Clearly, in this case, $\Lambda_{N, m}^{0}=\emptyset$. Denote

$$
\begin{aligned}
\left\|\tilde{G}_{\tau_{m}, 1}^{N_{m}}\right\|_{\tau_{m}, N_{m}}^{2}= & \left(1+\delta^{-1}\right) \| f\left(t, u_{m}^{N_{m}}(t), w_{m}(t), \mathcal{I}_{\tau_{m}, N_{m}} \frac{d}{d t} W_{m}(t)\right) \\
& -f\left(t, \mathcal{I}_{\tau_{m}, N_{m}} U_{m}(t), \mathcal{I}_{\tau_{m}, N_{m}} W_{m}(t), \mathcal{I}_{\tau_{m}, N_{m}} \frac{d}{d t} W_{m}(t)\right) \|_{\tau_{m}, N_{m}}^{2} .
\end{aligned}
$$

By (20) and the fact that $\sum_{k=0}^{N_{m}} \omega_{\tau_{m}, k}^{N_{m}}=\tau_{m}$, for any $\delta>0$ and $m>1$, we deduce that

$$
\begin{aligned}
\left\|G_{\tau_{m}, 1}^{N_{m}}\right\|_{\tau_{m}, N_{m}}^{2} \leq & (1+\delta)(1+\tilde{\delta}) r_{3}^{2}\left\|\frac{d}{d t} w_{m}-\frac{d}{d t} W_{m}\right\|_{\tau_{m}}^{2} \\
& +(1+\delta)\left(1+\tilde{\delta}^{-1}\right) r_{3}^{2}\left\|\frac{d}{d t} W_{m}-\mathcal{I}_{\tau_{m}, N_{m}} \frac{d}{d t} W_{m}\right\|_{\tau_{m}, N_{m}}^{2} \\
& +\left\|\tilde{G}_{\tau_{m}, 1}^{N_{m}}\right\|_{\tau_{m}, N_{m}}^{2},
\end{aligned}
$$

where $\left\|\frac{d}{d t} W_{m}-\mathcal{I}_{\tau_{m}, N_{m}} \frac{d}{d t} W_{m}\right\|_{\tau_{m}}^{2} \leq \sum_{j=1}^{m} c \hat{\tau}^{2 r-2} N^{2-2 r}\left|U_{j}\right|_{r, \tau_{j}}^{2} \leq c \hat{\tau}^{2 r-2} N^{2-2 r}|U|_{r, T_{m}}^{2}$ and

$$
\begin{aligned}
\left\|\frac{d}{d t} w_{m}-\frac{d}{d t} W_{m}\right\|_{\tau_{m}}^{2} & =\int_{0}^{\tau_{m}}\left[\frac{d}{d t} u^{N}\left(\theta\left(T_{m-1}+t\right)\right)-\frac{d}{d t} U\left(\theta\left(T_{m-1}+t\right)\right)\right]^{2} d t \\
& =\int_{T_{m-1}}^{T_{m}}\left[\frac{d}{d t} u^{N}(\theta(t))-\frac{d}{d t} U(\theta(t))\right]^{2} d t \\
& =\lambda \int_{\lambda T_{m-1}}^{\lambda T_{m}}\left[\frac{d}{d t} u^{N}(t)-\frac{d}{d t} U(t)\right]^{2} d t \\
& \leq \lambda \int_{0}^{T}\left[\frac{d}{d t} u^{N}(t)-\frac{d}{d t} U(t)\right]^{2} d t \\
& =\lambda \sum_{j=1}^{m-1}\left\|\frac{d}{d t} u_{j}^{N_{j}}-\frac{d}{d t} U_{j}\right\|_{\tau_{j}}^{2}+\lambda\left\|\frac{d}{d t} u_{m}^{N_{m}}-\frac{d}{d t} U_{m}\right\|_{\tau_{m}}^{2} \\
& \leq \lambda\left\|\frac{d}{d t} u_{m}^{N_{m}}-\frac{d}{d t} U_{m}\right\|_{\tau_{m}}^{2}+c \hat{\tau}^{2 r-2} N^{3-2 r}|U|_{r, T_{m}}^{2} .
\end{aligned}
$$

Due to (8),

$$
\begin{aligned}
\left\|G_{\tau_{m}, 1}^{N_{m}}\right\|_{\tau_{m}, N_{m}}^{2} \leq & (1+\delta)(1+\tilde{\delta})(1+\hat{\delta}) \lambda r_{3}^{2}\left\|\frac{d}{d t} E_{m}^{N_{m}}\right\|_{\tau_{m}}^{2} \\
& +c \hat{\tau}^{2 r-2} N^{3-2 r}|U|_{r, T_{m}}^{2}+\left\|\tilde{G}_{\tau_{m}, 1}^{N_{m}}\right\|_{\tau_{m}, N_{m}}^{2}
\end{aligned}
$$


By (31), we have

$$
\left\|\frac{d}{d t} E_{m}^{N_{m}}\right\|_{\tau_{m}, N_{m}}^{2} \leq \frac{\left(1+\delta_{0}\right)}{\hat{c}}\left\|\tilde{G}_{\tau_{m}, 1}^{N_{m}}\right\|_{\tau_{m}, N_{m}}^{2}+c \hat{\tau}^{2 r-2} N^{3-2 r}|U|_{r, T_{m}}^{2},
$$

where $\hat{c}=1-(1+\delta)(1+\tilde{\delta})(1+\hat{\delta}) \lambda r_{3}^{2}$. Applying Lemma 2 and (18)-(19), we deduce that

$$
\begin{aligned}
\left\|\tilde{G}_{\tau_{m}, 1}^{N_{m}}\right\|_{\tau_{m}, N_{m}}^{2} \leq & \left(1+\delta^{-1}\right)\left(1+\delta_{1}\right) 5 r_{1}^{2}\left\|E_{m}^{N_{m}}\right\|_{\tau_{m}}^{2} \\
& +\left(1+\delta^{-1}\right)\left(1+\delta_{1}^{-1}\right) 5 r_{2}^{2}\left\|w_{m}-\mathcal{I}_{\tau_{m}, N_{m}} W_{m}\right\|_{\tau_{m}}^{2},
\end{aligned}
$$

where

$$
\begin{aligned}
& \left\|w_{m}-\mathcal{I}_{\tau_{m}, N_{m}} W_{m}\right\|_{\tau_{m}}^{2} \\
& \quad \leq(1+\varepsilon)\left\|w_{m}-W_{m}\right\|_{\tau_{m}}^{2}+\left(1+\varepsilon^{-1}\right)\left\|W_{m}-\mathcal{I}_{\tau_{m}, N_{m}} W_{m}\right\|_{\tau_{m}}^{2} \\
& \quad \leq(1+\varepsilon) \lambda^{-1}\left\|u_{m}^{N_{m}}-U_{m}\right\|_{\tau_{m}}^{2}+c \varepsilon^{-1} \hat{\tau}^{2 r} N^{3-2 r}|U|_{r, T_{m}}^{2} .
\end{aligned}
$$

It can be found that

$$
\begin{aligned}
\left\|\frac{d}{d t} E_{m}^{N_{m}}\right\|_{\tau_{m}, N_{m}}^{2} \leq & \frac{\left(1+\delta_{0}\right)\left(1+\delta^{-1}\right)\left(1+\delta_{1}\right) 5 r_{1}^{2}}{\hat{c}}\left\|E_{m}^{N_{m}}\right\|_{\tau_{m}}^{2} \\
& +\frac{(1+\varepsilon)\left(1+\delta_{0}\right)\left(1+\delta^{-1}\right)\left(1+\delta_{1}^{-1}\right) 5 \lambda^{-1} r_{2}^{2}}{\hat{c}}\left\|u_{m}^{N_{m}}-U_{m}\right\|_{\tau_{m}}^{2} \\
& +c \varepsilon^{-1} \hat{\tau}^{2 r-2} N^{3-2 r}|U|_{r, T_{m}}^{2} .
\end{aligned}
$$

Applying Lemma 3, we deduce that

$$
\begin{aligned}
\frac{1}{2} \| & t^{-1}\left(E_{m}^{N_{m}}-E_{m}^{N_{m}}(0)\right) \|_{\tau_{m}}^{2}+\tau_{m}^{-1}\left|E_{m}^{N_{m}}\left(\tau_{m}\right)-E_{m}^{N_{m}}(0)\right|^{2} \\
\leq & \frac{2\left(1+\delta_{0}\right)\left(1+\delta^{-1}\right)\left(1+\delta_{1}\right) 5 r_{1}^{2}}{\hat{c}}\left\|E_{m}^{N_{m}}\right\|_{\tau_{m}}^{2} \\
& +\frac{2(1+\varepsilon)\left(1+\delta_{0}\right)\left(1+\delta^{-1}\right)\left(1+\delta_{1}^{-1}\right) 5 \lambda^{-1} r_{2}^{2}}{\hat{c}}\left\|u_{m}^{N_{m}}-U_{m}\right\|_{\tau_{m}}^{2} \\
& +c \varepsilon^{-1} \hat{\tau}^{2 r-2} N^{3-2 r}|U|_{r, T_{m}}^{2} .
\end{aligned}
$$

Thus, we have

$$
\begin{aligned}
\left(\frac{1}{2}-\right. & \frac{2\left(1+\delta_{0}\right)\left(1+\delta^{-1}\right)\left(1+\delta_{1}\right) 5 \tau_{m} r_{1}^{2}}{\hat{c}} \\
& \left.-\frac{2(1+\varepsilon)\left(1+\delta_{0}\right)\left(1+\delta^{-1}\right)\left(1+\delta_{1}^{-1}\right) 5 \tau_{m} \lambda^{-1} r_{2}^{2}}{\hat{c}}\right)\left\|u_{m}^{N_{m}}-U_{m}\right\|_{\tau_{m}}^{2} \\
& +(1+\varepsilon)^{2} \tau_{m}\left|E_{m}^{N_{m}}\left(\tau_{m}\right)-E_{m}^{N_{m}}(0)\right|^{2} \\
\leq & c \varepsilon^{-1} \hat{\tau}^{2 r} N^{3-2 r}|U|_{r, T_{m}}^{2}+c \varepsilon^{-1} \tau_{m}\left(E_{m}^{N_{m}}(0)\right)^{2} .
\end{aligned}
$$

Similar to the proof of Case 2.1, we complete the proof. 
Case 3.2 Consider (29) with the delay function $\theta(t)$ satisfying (22). The primary discontinuity points $t_{i}=i \tau \in[0, T]$ are contained in the set of $\left\{T_{k}\right\}$, then $\tau_{m} \leq \tau$. Moreover, $f(t, x, y, z)$ satisfies the Lipschitz condition (17), but $r_{3}$ satisfies $0 \leq r_{3}<1$.

Theorem 5 Consider the $N D D E(1)$, where $f(t, x, y, z)$ is $C^{r}$-continuous in $[0, T] \times R^{d} \times$ $R^{d} \times R^{d}$, and the initial function $V(t)$ is $C^{r}$-continuous. If the conditions (18)-(20) and (22) hold, $U \in H^{r}(0, T)$ with $2 \leq r \leq N+1,(1+\delta) 5 \tau_{m}^{2} r_{1}^{2} \leq \beta_{1}<\frac{1}{4}$, and

$$
\frac{\left(1+\delta_{0}\right)\left(1+\delta^{-1}\right) 5 \tau_{m}^{2}\left(\left(1+\delta_{1}\right) r_{1}^{2}+\left(1+\delta_{1}^{-1}\right) r_{2}^{2}\right)}{1-\left(1+\delta_{0}\right)(1+\delta)(1+\hat{\delta})(1+\tilde{\delta}) r_{3}^{2}} \leq \beta_{2}<\frac{1}{4},
$$

for certain $\delta, \delta_{0}, \delta_{1}, \hat{\delta}, \tilde{\delta}>0$, then, for any $1 \leq m \leq M$, we have

$$
\begin{aligned}
& \left\|U-u^{N}\right\|_{L_{\left(T_{m-1}, T_{m}\right)}^{2}}^{2} \leq c_{\beta_{1}, \beta_{2}} \hat{\tau}^{2 r} N^{3-2 r}|U|_{r, T_{m}}^{2}, \\
& \left|U\left(\tau_{m}\right)-u^{N}\left(\tau_{m}\right)\right|^{2} \leq c_{\beta_{1}, \beta_{2}} \hat{\tau}^{2 r-1} N^{3-2 r}|U|_{r, T_{m}}^{2} .
\end{aligned}
$$

In particular, $\max _{t \in\left[T_{m-1}, T_{m}\right]}\left|U(t)-u^{N}(t)\right|^{2} \leq c_{\beta_{1}, \beta_{2}} \hat{\tau}^{2 r-1} N^{3-2 r}|U|_{r, T_{m}}^{2}$, where $c_{\beta_{1}, \beta_{2}}$ is a positive constant depending only on $\beta_{1}, \beta_{2}$.

Proof (I) If $\tau \geq T_{m}$, then $\theta\left(T_{m-1}+t\right)<0, t \in\left[0, \tau_{m}\right]$. In this case, $\Lambda_{N, m}^{j}=\emptyset(1 \leq j \leq m)$. Thus, $\left\|G_{\tau_{m}, 1}^{N_{m}}\right\|_{\tau_{m}, N_{m}}^{2} \leq r_{1}^{2}\left\|E_{m}^{N_{m}}\right\|_{\tau_{m}, N_{m}}^{2}$. Therefore, we have

$$
\begin{aligned}
& \left(\frac{1}{2}-2(1+\varepsilon)(1+\delta) 5 \tau_{m}^{2} r_{1}^{2}\right)\left\|E_{m}^{N_{m}}\right\|_{\tau_{m}}^{2}+(1+\varepsilon) \tau_{m}\left|E_{m}^{N_{m}}\left(\tau_{m}\right)-E_{m}^{N_{m}}(0)\right|^{2} \\
& \quad \leq c \varepsilon^{-1} \hat{\tau}^{2 r} N^{3-2 r}|U|_{r, T_{m}}^{2}+c \varepsilon^{-1} \tau_{m}\left(E_{m}^{N_{m}}(0)\right)^{2} .
\end{aligned}
$$

Let $\varepsilon=1 / \beta_{1}$, then $(1+\varepsilon)(1+\delta) 5 \tau_{m}^{2} r_{1}^{2} \leq \beta_{1}<1 / 4$. We deduce that

$$
\begin{aligned}
& \left\|u_{m}^{N_{m}}-U_{m}\right\|_{\tau_{m}}^{2} \leq c_{\beta_{1}} \hat{\tau}^{2 r} N^{3-2 r}|U|_{r, T_{m}}^{2}, \\
& \left|U_{m}\left(\tau_{m}\right)-u_{m}^{N}\left(\tau_{m}\right)\right|^{2} \leq c_{\beta_{1}} \hat{\tau}^{2 r-1} N^{3-2 r}|U|_{r, T_{m}}^{2}, \\
& \max _{t \in\left[0, \tau_{m}\right]}\left|U_{m}(t)-u_{m}^{N}(t)\right|^{2} \leq c_{\beta_{1}} \hat{\tau}^{2 r-1} N^{3-2 r}|U|_{r, T_{m}}^{2},
\end{aligned}
$$

where $c_{\beta_{1}}=\frac{c \varepsilon^{-1}}{\frac{1}{2}-2(1+\varepsilon)(1+\delta) 5 \tau_{m}^{2} r_{1}^{2}}$.

(II) If $\tau \leq T_{m-1}$, then $\theta\left(T_{m-1}+t\right)=T_{m-1}-\tau+t \geq 0, t \in\left[0, \tau_{m}\right]$. For $\tau_{m} \leq \tau, \theta\left(T_{m-1}+t\right) \leq$ $T_{m-1}$. In this case, $\Lambda_{N, m}^{0}=\emptyset$ and $\Lambda_{N, m}^{m}=\emptyset$. The proof is similar to Theorem 4; we can deduce that $\left\|w_{m}-W_{m}\right\|_{\tau_{m}}^{2} \leq\left\|u_{m}^{N_{m}}-U_{m}\right\|_{\tau_{m}}^{2}+c \hat{\tau}^{2 r} N^{3-2 r}|U|_{r, T_{m}}^{2}$ and

$$
\begin{aligned}
\left\|\frac{d}{d t} w_{m}-\frac{d}{d t} W_{m}\right\|_{\tau_{m}}^{2} & =\int_{T_{m-1}}^{T_{m}}\left[\frac{d}{d t} u^{N}(\theta(t))-\frac{d}{d t} U(\theta(t))\right]^{2} d t \\
& =\int_{T_{m-1}-\tau}^{T_{m}-\tau}\left[\frac{d}{d t} u^{N}(t)-\frac{d}{d t} U(t)\right]^{2} d t \\
& \leq \int_{0}^{T_{m-1}}\left[\frac{d}{d t} u^{N}(t)-\frac{d}{d t} U(t)\right]^{2} d t \leq c \hat{\tau}^{2 r-2} N^{3-2 r}|U|_{r, T_{m}}^{2} .
\end{aligned}
$$


Then we can obtain

$$
\begin{aligned}
& \left\|u_{m}^{N_{m}}-U_{m}\right\|_{\tau_{m}}^{2} \leq c_{\beta_{2}} \hat{\tau}^{2 r} N^{3-2 r}|U|_{r, T_{m}}^{2}, \\
& \left|U_{m}\left(\tau_{m}\right)-u_{m}^{N}\left(\tau_{m}\right)\right|^{2} \leq c_{\beta_{2}} \hat{\tau}^{2 r-1} N^{3-2 r}|U|_{r, T_{m}}^{2}, \\
& \max _{t \in\left[0, \tau_{m}\right]}\left|U_{m}(t)-u_{m}^{N}(t)\right|^{2} \leq c_{\beta_{2}} \hat{\tau}^{2 r-1} N^{3-2 r}|U|_{r, T_{m}}^{2} .
\end{aligned}
$$

Case 3.3 Assume that the delay function $\theta(t)$ satisfies (24) and the function $f(t, x, y, z)$ satisfies the Lipschitz conditions (18)-(20), but $r_{3}$ satisfies $0 \leq r_{3}<\frac{1}{\sqrt{c_{0}}}$, where $c_{0}=$ $\max _{t \in[0, T]} \frac{1}{\left[\theta^{-1}(t)\right]^{\prime}}$.

Theorem 6 Consider the $N D D E(1)$, where $f(t, x, y, z)$ is $C^{r}$-continuous in $[0, T] \times R^{d} \times$ $R^{d} \times R^{d}$, and the initial function $V(t)$ is $C^{r}$-continuous. If the conditions (18)-(20) and (24) hold, $U \in H^{r}(0, T)$ with integer $2 \leq r \leq N+1, c_{1}=\min _{t \in[0, T]} \frac{1}{\left[\theta^{-1}(t)\right]^{\prime}},(1+\delta) 5 \tau_{m}^{2} r_{1}^{2} \leq \beta_{1}<\frac{1}{4}$, and, for certain $\delta, \delta_{0}, \delta_{1}, \hat{\delta}, \tilde{\delta}>0$,

$$
\begin{aligned}
& \frac{\left(1+\delta_{0}\right)\left(1+\delta^{-1}\right)\left(1+\delta_{1}\right) 5 \tau_{m}^{2} r_{1}^{2}+\left(1+\delta_{0}\right)\left(1+\delta^{-1}\right)\left(1+\delta_{1}^{-1}\right) 5 \tau_{m}^{2} c_{1} r_{2}^{2}}{1-\left(1+\delta_{0}\right)(1+\delta)(1+\hat{\delta})(1+\tilde{\delta}) c_{0} r_{3}^{2}} \\
& \quad \leq \beta_{2}<\frac{1}{4}
\end{aligned}
$$

then we have (36). In particular, $\max _{t \in\left[T_{m-1}, T_{m}\right]}\left|U(t)-u^{N}(t)\right|^{2} \leq c_{\beta_{1}, \beta_{2}} \hat{\tau}^{2 r-1} N^{3-2 r}|U|_{r, T_{m}}^{2}$, where $c_{\beta_{1}, \beta_{2}}$ is a positive constant depending only on $\beta_{1}, \beta_{2}$.

Proof Due to (24), we can find $t_{0}>0$ which satisfies $\theta\left(t_{0}\right)=0$.

(I) If $t_{0} \geq T_{m}$, then $\theta\left(T_{m-1}+t\right)<0$. Obviously, $\Lambda_{N, m}^{j}=\emptyset(1 \leq j \leq m)$, the proof is similar to $\tau \geq T_{m}$ in Case 3.2. Therefore, we can obtain

$$
\begin{aligned}
& \left\|u_{m}^{N_{m}}-U_{m}\right\|_{\tau_{m}}^{2} \leq c_{\beta_{1}} \hat{\tau}^{2 r} N^{3-2 r}|U|_{r, T_{m}}^{2}, \\
& \left|U_{m}\left(\tau_{m}\right)-u_{m}^{N}\left(\tau_{m}\right)\right|^{2} \leq c_{\beta_{1}} \hat{\tau}^{2 r-1} N^{3-2 r}|U|_{r, T_{m}}^{2}, \\
& \max _{t \in\left[0, \tau_{m}\right]}\left|U_{m}(t)-u_{m}^{N}(t)\right|^{2} \leq c_{\beta_{1}} \hat{\tau}^{2 r-1} N^{3-2 r}|U|_{r, T_{m}}^{2} .
\end{aligned}
$$

(II) If $t_{0} \leq T_{m-1}$, then $\theta\left(T_{m-1}+t\right) \geq 0$ for $t \in\left[0, \tau_{m}\right]$. In this case, $\Lambda_{N, m}^{0}=\emptyset$. The proof is similar to $\tau \leq T_{m-1}$ in Case 3.2, where $\left\|w_{m}-W_{m}\right\|_{\tau_{m}}^{2} \leq c_{1}\left\|u_{m}^{N_{m}}-U_{m}\right\|_{\tau_{m}}^{2}+c \hat{\tau}^{2 r} N^{3-2 r}|U|_{r, T_{m}}^{2}$ and

$$
\begin{aligned}
\left\|\frac{d}{d t} w_{m}-\frac{d}{d t} W_{m}\right\|_{\tau_{m}}^{2} & =\int_{0}^{\tau_{m}}\left[\frac{d}{d t} u^{N}\left(\theta\left(T_{m-1}+t\right)\right)-\frac{d}{d t} U\left(T_{m-1}+t\right)\right]^{2} d t \\
& \leq c_{0}\left\|\frac{d}{d t} u_{m}^{N_{m}}-\frac{d}{d t} U_{m}\right\|_{\tau_{m}}^{2}+c \hat{\tau}^{2 r-2} N^{3-2 r}|U|_{r, T_{m}}^{2} .
\end{aligned}
$$

Thus, we have

$$
\begin{aligned}
& \left\|u_{m}^{N_{m}}-U_{m}\right\|_{\tau_{m}}^{2} \leq c_{\beta_{2}} \hat{\tau}^{2 r} N^{3-2 r}|U|_{r, T_{m}}^{2}, \\
& \left|U_{m}\left(\tau_{m}\right)-u_{m}^{N}\left(\tau_{m}\right)\right|^{2} \leq c_{\beta_{2}} \hat{\tau}^{2 r-1} N^{3-2 r}|U|_{r, T_{m}}^{2} \\
& \max _{t \in\left[0, \tau_{m}\right]}\left|U_{m}(t)-u_{m}^{N}(t)\right|^{2} \leq c_{\beta_{2}} \hat{\tau}^{2 r-1} N^{3-2 r}|U|_{r, T_{m}}^{2} .
\end{aligned}
$$


(III) If $T_{m-1}<t_{0} \leq T_{m}$, then we decompose the interval $\left[0, \tau_{m}\right]$ into $\left[0, t_{0}-T_{m-1}\right]$ and $\left[t_{0}-T_{m-1}, \tau_{m}\right]$. Denote $\tau_{m, 1}=t_{0}-T_{m-1}$ and $\tau_{m, 2}=\tau_{m}-\left(t_{0}-T_{m-1}\right)$, we shall use $u_{m, 1}^{N_{m}} \in$ $\mathcal{P}_{N_{m}+1}\left(0, \tau_{m, 1}\right)$ and $u_{m, 2}^{N_{m}} \in \mathcal{P}_{N_{m}+1}\left(0, \tau_{m, 2}\right)$ to approximate the solution $U$ in the subinterval $\left[0, \tau_{m, 1}\right]$ and $\left[\tau_{m, 1}, \tau_{m}\right]$, respectively.

The global numerical solution of (1) is given by

$$
\begin{aligned}
& u^{N}\left(T_{m-1}+t\right)=u_{m}^{N_{m}}(t)=u_{m, 1}^{N_{m}}(t), \quad 0 \leq t \leq \tau_{m, 1}, \\
& u^{N}\left(T_{m-1}+\tau_{m, 1}+t\right)=u_{m}^{N_{m}}\left(\tau_{m, 1}+t\right)=u_{m, 2}^{N_{m}}(t), \quad 0 \leq t \leq \tau_{m, 2} .
\end{aligned}
$$

Let $U_{m, 1}(t)=U_{m}(t)=U\left(T_{m-1}+t\right)$ for $t \in\left[0, \tau_{m, 1}\right]$ and $U_{m, 2}(t)=U_{m}\left(\tau_{m, 1}+t\right)=U\left(T_{m-1}+\right.$ $\left.\tau_{m, 1}+t\right)$ for $t \in\left[0, \tau_{m, 2}\right]$.

Firstly, we seek $u_{m, 1}^{N_{m}} \in \mathcal{P}_{N+1}\left(0, \tau_{m, 1}\right)$. Obviously, $\Lambda_{N, m}^{j}=\emptyset(1 \leq j \leq m)$. The proof is similar to the case of $t_{0}>T_{m}$, and we can obtain the results that

$$
\begin{aligned}
& \left\|u_{m, 1}^{N_{m}}-U_{m, 1}\right\|_{\tau_{m}}^{2} \leq c_{\beta_{1}} \hat{\tau}^{2 r} N^{3-2 r}|U|_{r, T_{m}}^{2}, \\
& \left|U_{m, 1}\left(\tau_{m, 1}\right)-u_{m, 1}^{N}\left(\tau_{m, 1}\right)\right|^{2} \leq c_{\beta_{1}} \hat{\tau}^{2 r-1} N^{3-2 r}|U|_{r, T_{m}}^{2}, \\
& \max _{t \in\left[0, \tau_{m, 1}\right]}\left|U_{m, 1}(t)-u_{m, 1}^{N}(t)\right|^{2} \leq c_{\beta_{1}} \hat{\tau}^{2 r-1} N^{3-2 r}|U|_{r, T_{m}}^{2} .
\end{aligned}
$$

Then we evaluate $u_{m, 2}^{N_{m}} \in \mathcal{P}_{N_{m+1}}\left(0, \tau_{m, 2}\right)$. Obviously, $\Lambda_{N, m}^{0}=\emptyset$, the proof is similar to $t_{0}<$ $T_{m-1}$. Therefore, we can obtain

$$
\begin{aligned}
& \left\|u_{m, 2}^{N_{m}}-U_{m, 2}\right\|_{\tau_{m, 2}}^{2} \leq c_{\beta_{2}} \hat{\tau}^{2 r} N^{3-2 r}|U|_{r, T_{m}}^{2}, \\
& \left|U_{m, 2}\left(\tau_{m, 2}\right)-u_{m, 2}^{N}\left(\tau_{m, 2}\right)\right|^{2} \leq c_{\beta_{2}} \hat{\tau}^{2 r-1} N^{3-2 r}|U|_{r, T_{m}}^{2}, \\
& \max _{t \in\left[0, \tau_{m, 2}\right]}\left|U_{m, 2}(t)-u_{m, 2}^{N}(t)\right|^{2} \leq c_{\beta_{2}} \hat{\tau}^{2 r-1} N^{3-2 r}|U|_{r, T_{m}}^{2} .
\end{aligned}
$$

Thus, we can obtain the results of Theorem 6 .

\subsection{Numerical results}

In this subsection, we give some numerical results to illustrate the efficiency of our multiple-domain algorithm.

Example 4 Consider (25) by multiple-domain algorithm. Firstly, we decompose the interval $[0, T]$ with $T=0.5$ into two intervals with uniform $\tau_{m}=0.25$ and $N_{m}=N, m=1,2$. We plot the numerical errors at $t=0.5$ in Figure 3, which indicates that the numerical errors decay exponentially as $N$ increases. Then we consider (25) with $T=1$ and decompose equally the interval $[0, T]$ into $M=1,2$ and 4 subintervals, respectively. The inequality (33) is satisfied for $M=4$, but for $M=2$ and $M=1$, the inequality (33) is no longer valid. Figure 4 indicates that the numerical errors decay exponentially as $N_{m}=N$ increases and $\tau_{m}$ decreases, $m=1, \ldots, M$. In particular, it can be observed from Figure 4 that even if the condition (33) is not satisfied, our multiple-domain algorithm is still valid (see the cases of $M=1,2)$.

Example 5 Consider (26) by multiple-domain algorithm. In Figure 5, we plot the numerical errors at $t=T=0.5$ using the multiple-domain scheme (29) with uniform $\tau_{m}=0.25$ and $N_{m}=N, m=1,2$. It indicates that the numerical errors decay exponentially as $N$ increases. 


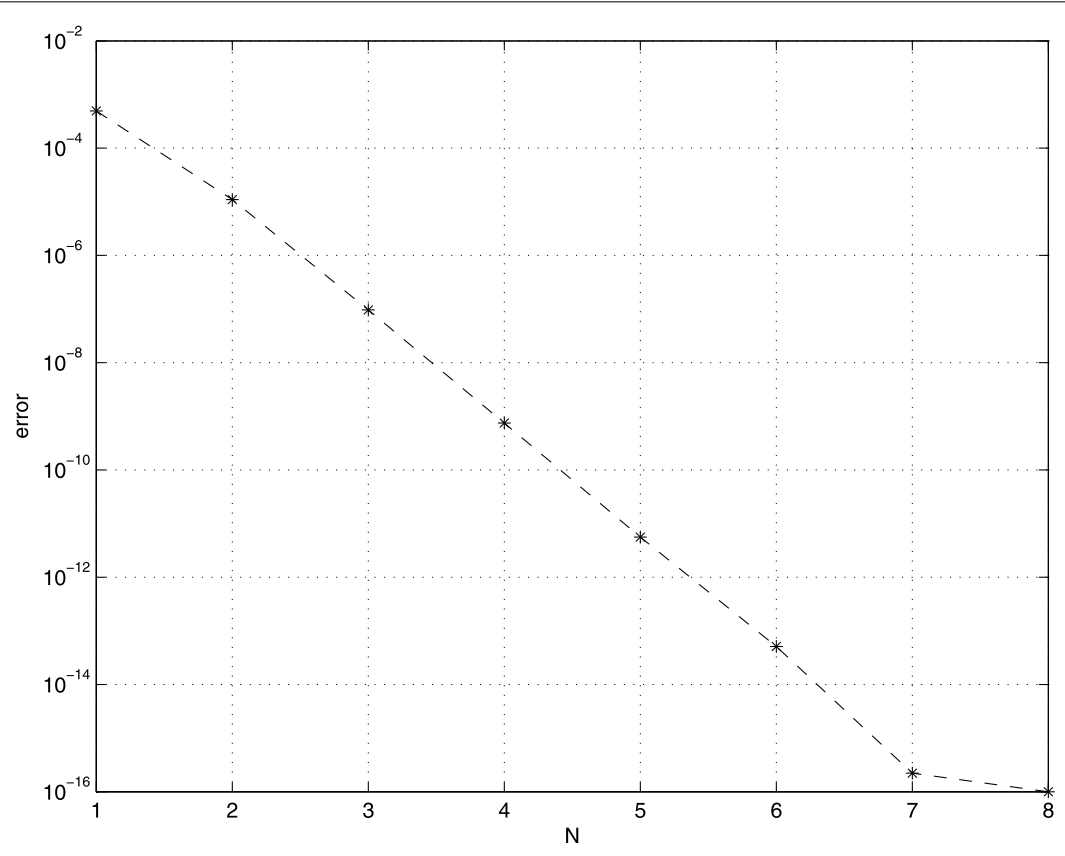

Figure 3 The numerical errors of (25) at $t=T=0.5$ with uniform $\tau_{m}=0.25, m=1,2$.

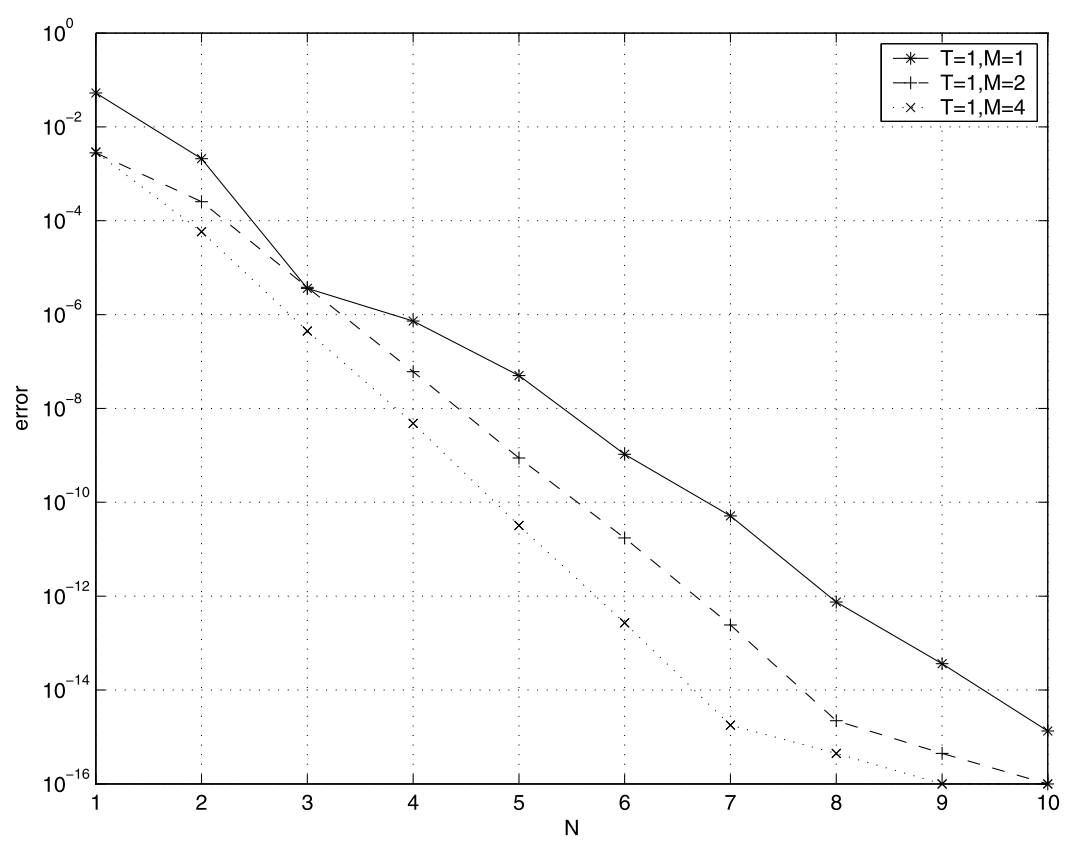

Figure 4 The numerical errors of (25) for different $M$. 


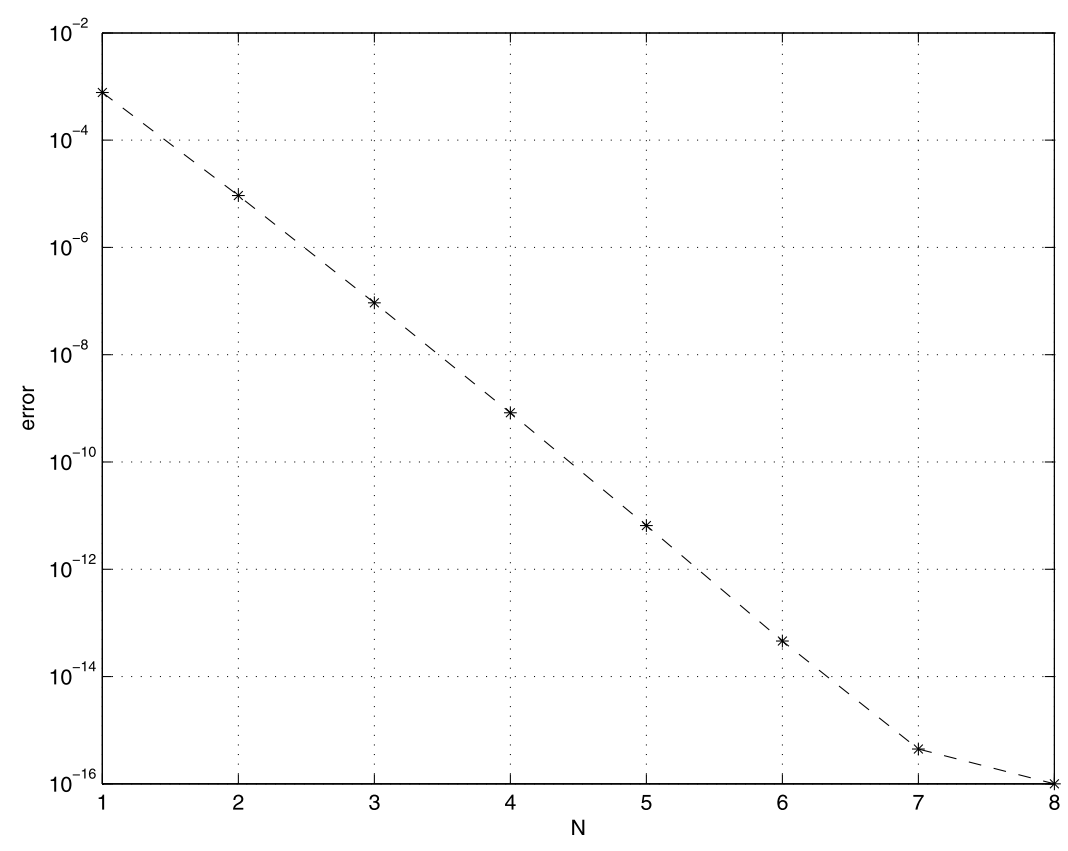

Figure 5 The numerical errors of (26) or (27) at $t=T=0.5$ with uniform $\tau_{m}=0.25, m=1,2$.

Example 6 Consider (27) by multiple-domain algorithm. To consider (27), we use multiple-domain method at $t=T=0.5$ with uniform $\tau_{m}=0.25$ and $N_{m}=N, m=1,2$. The multiple-domain discrete scheme of (27) is the same as (26). So, we can also see the convergence of the numerical solution of this equation in Figure 5.

Example 7 Consider the following equation:

$$
\left\{\begin{array}{l}
\frac{d}{d t} u(t)=-u^{\prime}(t-1), \quad 0 \leq t \leq 3 \\
u(t)=t, \quad-1 \leq t \leq 0 \\
u(0)=0
\end{array}\right.
$$

The exact solution is

$$
u(t)= \begin{cases}-t, & 0 \leq t \leq 1 \\ t-2, & 1 \leq t \leq 2 \\ 2-t, & 2 \leq t \leq 2\end{cases}
$$

where $0,1,2,3$ are primary discontinuity points. To consider the equation (38), we use multiple-domain method at $T=3$ with uniform $\tau_{m} \equiv 1$ and $N_{m}=N(m=1,2,3)$. For the linear property of (39), the approximate solution is equal to (39) on each subinterval. Therefore, we find that the values of the numerical error function are all zero at point $t=1,2,3$ with $N_{m}=1, \ldots, 5(m=1,2,3)$. So, the multiple-domain method is efficient and accurate.

\section{Conclusions}

The single-step and multiple-domain Legendre-Gauss collocation methods are proposed for nonlinear NDDEs in this article. These approaches have two attractive features. 
(I) We can use moderate $N$ to evaluate the numerical solutions more effectively by using the multiple-domain Legendre-Gauss collocation method. We benefit from the orthogonality of Legendre polynomials, while in the derivation of algorithm of the implicit RungeKutta method, one used the Lagrange interpolation on the Legendre-Gauss interpolation nodes, which is not stable for large $N$. In particular, our methods are much easier to be implemented than the implicit Runge-Kutta method for NDDEs, since we only need to save the coefficients of numerical solutions in each step.

(II) The numerical errors of our methods are characterized by the semi-norms of exact solutions in certain Sobolev spaces. These sharp norms are in particular necessary for the problems with degenerate initial data.

The numerical results demonstrate the spectral accuracy of proposed algorithms and coincide with the theoretical analysis very well.

Competing interests

The authors declare that they have no competing interests.

Authors' contributions

All authors contributed equally to the writing of this paper. All authors read and approved the final manuscript.

\section{Acknowledgements}

The authors would like to thank the anonymous referees for their valuable comments, which helped us to improve the present paper. This work was supported by the National Natural Science Foundation of China $(11101109,11271102)$, the Natural Science Foundation of Hei-long-jiang Province of China (A201107), PIRS of HIT (A201405) and SRF for ROCS, SEM.

Received: 12 September 2014 Accepted: 25 December 2014 Published online: 30 January 2015

References

1. Weldom, TE, Kirk, J, Finlay, HM: Cyclical granulopoiesis in chronic granulocytic leukemia: a simulation study. Blood 43, 379-387 (1974)

2. Brayton, RK: Small signal stability criterion for networks containing lossless transmission lines. IBM J. Res. Dev. 121, 431-440 (1968)

3. Kuang, Y: Delay Differential Equations with Application in Population Dynamics. Academic Press, Boston (1993)

4. Ruehli, AE, Miekkala, U, Bellen, A, Heeb, H: Stable time domain solutions for EMC problems using PEEC circuit models. In: Proceedings of IEEE Int. Symposium on Electromagnetic Compatibility (1994)

5. Guglielmi, N: Inexact Newton methods for the steady-state analysis of nonlinear circuits. Math. Models Methods Appl. Sci. 6, 43-57 (1996)

6. Hale, JK, Verduyn Lunel, SM: Introduction to Functional Differential Equations. Springer, New York (1993)

7. Liu, Y: Numerical solution of implicit neutral functional differential equations. SIAM J. Numer. Anal. 36, 516-528 (1999)

8. Bellen, A, Guglielmi, N, Zennaro, M: On the contractivity and asymptotic stability of systems of delay differential equations of neutral type. BIT Numer. Math. 39, 1-24 (1999)

9. Vermiglio, R, Torelli, L: A stable numerical approach for implicit non-linear neutral delay differential equations. BIT Numer. Math. 43, 195-215 (2003)

10. Sun, LP: Stability analysis for delay differential equations with multidelays and numerical examples. Math. Comput. 75, 151-165 (2005)

11. Ito, K, Tran, HT, Manitius, A: A fully-discrete spectral method for delay-differential equations. SIAM J. Numer. Anal. 28, $1121-1140$ (1991)

12. Guo, BY, Wang, ZQ: Legendre-Gauss collocation methods for ordinary differential equations. Adv. Comput. Math. 30 249-280 (2009)

13. Guo, BY, Yan, JP: Legendre-Gauss collocation methods for initial value problems of second ordinary differential equations. Appl. Numer. Math. 59, 1386-1408 (2009)

14. Wang, ZQ, Wang, LL: A Legendre-Gauss collocation method for nonlinear delay differential equations. Discrete Contin. Dyn. Syst., Ser. B 13, 685-708 (2010)

15. Wang, ZQ, Guo, BY: Legendre-Gauss-Radau collocation method for solving initial value problems of first order ordinary differential equations. J. Sci. Comput. 52, 226-255 (2012)

16. Bellen, A, Zennaro, M: Numerical Methods for Delay Differential Equations. Clarendon, Oxford (2005)

17. Adams, RA: Sobolev Spaces. Academic Press, New York (1975) 